\title{
Recent progress and open problems in the Bergman space
}

\author{
Alexandru Aleman, Håkan Hedenmalm, Stefan Richter \\ at Lund, Stockholm, and Knoxville
}

\section{An initial remark}

The following text is a modified and updated version of the problem collection [39], which was written in 1993 but became publicly available only in 1995. It was a survey of various open problems; a general survey of the field was provided in [41, 42] in 1998, written in 1995 and 1996, respectively. Since then, a number of new developments have taken place, which in their turn have led to new questions. We feel it is time to update the problem collection.

\section{The basic project}

Let $L_{a}^{2}(\mathbb{D})$ be the usual Bergman space of square area integrable analytic functions on the open unit disk $\mathbb{D}$, with norm

$$
\|f\|_{L^{2}}=\left(\int_{\mathbb{D}}|f(z)|^{2} d S(z)\right)^{1 / 2} .
$$

Here, $d S$ denotes area measure in $\mathbb{C}$, normalized by a constant factor:

$$
d S(z)=d x d y / \pi, \quad z=x+i y .
$$

A closed subspace $J$ of $L_{a}^{2}(\mathbb{D})$ is said to be $z$-invariant, or simply invariant, provided the product $z f$ belongs to $J$ whenever $f \in J$. Here, we use the standard notation $z$ for the coordinate function:

$$
z(\lambda)=\lambda, \quad \lambda \in \mathbb{D} .
$$

A sequence $A=\left\{a_{j}\right\}_{j}$ of points in $\mathbb{D}$, is said to be an $L_{a}^{2}(\mathbb{D})$ zero sequence if there exists a function in $L_{a}^{2}(\mathbb{D})$ that vanishes precisely on the sequence $A$, counting multiplicities.

Three important projects for this space are as follows.

PROBLEM 1 Characterize the invariant subspaces of $L_{a}^{2}(\mathbb{D})$.

PROBLEM 2 Characterize the $L_{a}^{2}(\mathbb{D})$ zero sequences.

PROBLEM 3 Find an effective factorization of the functions in $L_{a}^{2}(\mathbb{D})$.

By an effective factorization we mean one that is in some sense equivalent to that of the Hardy spaces, where Blaschke products, singular inner functions, and outer functions are involved.

Of the above three problems, the second and third ones are more likely to find definite answer than the first one. In fact, from one point of view, Problem 1 is as difficult as the famous invariant subspace problem in Hilbert space. Indeed, it is shown [10] how to apply the the dilation theory of Apostol, Bercovici, Foiass, and Pearcy [12] to obtain the following: If we could show that given two $z$-invariant subspaces $I, J$ in $L_{a}^{2}(\mathbb{D})$, with $I \subset J$, and $\operatorname{dim}(J \ominus I)=+\infty$, there exists another invariant subspace $K$, other than 
$I$ and $J$, but contained in $J$ and containing $I$, then every bounded linear operator on a separate Hilbert space must have a nontrivial invariant subspace. And it is understood that the invariant subspace problem for Hilbert space is really difficult. However, there are plenty of more reasonable subquestions regarding the invariant subspace lattice for $L_{a}^{2}(\mathbb{D})$. For instance, we might be better able to characterize an invariant subspace if we know something about its so-called weak spectrum (see [36, 63] for a definition). As an example of this, we mention that Aharon Atzmon [14, 15] has obtained a complete description of invariant subspaces in $L_{a}^{2}(\mathbb{D})$ with one-point spectra, also in wide classes of radially weighted Bergman spaces on $\mathbb{D}$. Another question which is tractable is to ask for a description of the maximal invariant subspaces in $L_{a}^{2}(\mathbb{D})$; see Section 10 for details.

There has been some progress on Problem 2. Charles Horowitz [52] obtained several interesting results. For instance, he proved that there are $L_{a}^{2}(\mathbb{D})$ zero sequences $A=\left\{a_{j}\right\}_{j}$ of non-Blaschke type, that is, having

$$
\sum_{j}\left(1-\left|a_{j}\right|\right)=+\infty
$$

and that every subsequence of a zero sequence is a zero sequence as well. He also showed that the union of two zero sequences for $L_{a}^{2}(\mathbb{D})$ need not be a zero sequence. Another important feature of the zero sequences that was apparently known before Horowitz' work is the angular dependency: inside any given Stolz angle, the zero set must meet the Blaschke condition, although it does not have to be met globally. Boris Korenblum [55] (see also [56]) found a characterization of the zero sequences for the larger topological vector space $A^{-\infty}$ of holomorphic functions $f$ in $\mathbb{D}$ with the growth bound

$$
|f(z)| \leq \frac{C(f)}{(1-|z|)^{N}}, \quad z \in \mathbb{D},
$$

for some positive real number $N$, and a positive constant $C(f)$ that may depend on the given function $f$. The description is in terms of Blaschke sums over star domains formed as unions of Stolz angles, as compared with the logarithmic entropy of the collection of the vertices of the Stolz angles on the unit circle. As a step toward the characterization of the zero sequences for $A^{-\infty}$, Korenblum obtains estimates which apply to the Bergman space $L_{a}^{2}(\mathbb{D})$, but there is a substantial gap in the constants, which cannot be brought down to be smaller than a factor of 2 with his methods. In another vein, Emile LeBlanc [58] and Gregory Bomash [18] obtained probabilistic conditions on zero sets. Using Korenblum's description of zero sequences, plus an added improvement in the constants, Kristian Seip [66] obtained a complete description of sampling and interpolating sequences, by realizing the interpolation implies "uniform zero sequence under Möbius translations", whereas sampling means "uniform non-zero sequence under Möbius translations". By sharpening his methods, Seip later obtained a description of the zero sequences for $L_{a}^{2}(\mathbb{D})$ of Korenblum type, where the gap in the constants that Korenblum had was gone, but a gap a smaller degree of magnitude remained [67]. A complete characterization of the zero sequences for $L_{a}^{2}(\mathbb{D})$ remains elusive. A different approach to this theorem of Seip is supplied in the book of Hedenmalm, Korenblum, and Zhu [45, Ch. 4].

As for Problem 3, the method of extremal functions, or in other words, inner divisors, has met with great success. The inner divisors constitute a modification of the classical inner functions from the Hardy space theory.

DEFINITION 2.1 A function $\varphi \in L_{a}^{2}(\mathbb{D})$ is said to be an inner divisor for $L_{a}^{2}(\mathbb{D})$ if

$$
h(0)=\int_{\mathbb{D}} h(z)|\varphi(z)|^{2} d S(z)
$$

holds for all bounded harmonic functions $h$ on $\mathbb{D}$. 
We note here that if normalized area measure $d S$ on $\mathbb{D}$ is replaced by normalized arc length measure in the above definition, we have a rather unusual, though equivalent, definition of the concept of an inner function in $H^{2}(\mathbb{D})$. The Hardy space $H^{2}(\mathbb{D})$ consists by definition of all analytic functions $f$ in the unit disk $\mathbb{D}$ satisfying

$$
\|f\|_{H^{2}}=\left(\sup _{0<r<1} \int_{-\pi}^{\pi}\left|f\left(r e^{i \theta}\right)\right|^{2} \frac{d \theta}{2 \pi}\right)^{1 / 2}<+\infty .
$$

In analogy with finite Blaschke products, we define the finite zero divisors as follows.

DEFINITION 2.2 An inner divisor for $L_{a}^{2}(\mathbb{D})$ is said to be a finite zero divisor for $L_{a}^{2}(\mathbb{D})$ if it extends continuously to the closed unit disk $\overline{\mathbb{D}}$. If $A$ is its finite zero set in $\mathbb{D}$, counting multiplicities, we shall denote this function by $\varphi_{A}$.

It is implicit in the above definition that a finite zero divisor for $L_{a}^{2}(\mathbb{D})$ only has finitely many zeros in $\mathbb{D}$, and that it is determined uniquely, up to a unimodular constant multiple, by its finite sequence of zeros. These facts may be derived from the results obtained in Hedenmalm's paper [33]. It is, moreover, known that to every finite sequence $A$ in $\mathbb{D}$, there exists a finite zero divisor $G_{A}$ vanishing precisely on $A$ inside $\mathbb{D}$.

DEFINITION 2.3 An inner divisor $\varphi$ is said to be a zero divisor for $L_{a}^{2}(\mathbb{D})$ if it is the limit (as $N \rightarrow+\infty$ ), in the topology of uniform convergence on compact subsets of $\mathbb{D}$, of a sequence of finite zero divisors $\varphi_{A_{N}}$, with $A_{1} \subset A_{2} \subset A_{3} \subset \ldots \mathbb{D}$.

We note in passing that a zero divisor for $L_{a}^{2}(\mathbb{D})$ is uniquely determined, up to multiplication by a unimodular constant factor, by its sequence of zeros, counting multiplicities. We shall frequently write $G_{A}$ for the zero divisor associated with the zero sequence $A$.

Given an inner divisor $\varphi$ for $L_{a}^{2}(\mathbb{D})$, we denote by $\Phi_{\varphi}$ the (potential) function

$$
\Phi_{\varphi}(z)=\int_{\mathbb{D}} G(z, \zeta)\left(|\varphi(\zeta)|^{2}-1\right) d S(\zeta), \quad z \in \mathbb{D}
$$

here, $G(z, \zeta)$ stands for the Green function for the Laplacian $\Delta$ :

$$
G(z, \zeta)=\log \left|\frac{\zeta-z}{1-\bar{\zeta} z}\right|^{2}, \quad(z, \zeta) \in \mathbb{D}^{2} .
$$

Throughout this paper, we use the slightly nonstandard Laplacian

$$
\Delta_{z}=\frac{1}{4}\left(\frac{\partial^{2}}{\partial x^{2}}+\frac{\partial^{2}}{\partial y^{2}}\right), \quad z=x+i y,
$$

and we regard locally integrable functions $u$ on $\mathbb{D}$ as distributions via the dual action

$$
\langle u, f\rangle=\int_{\mathbb{D}} u(z) f(z) d S(z),
$$

for test functions $f$. The function $\Phi_{\varphi}$ solves the boundary value problem

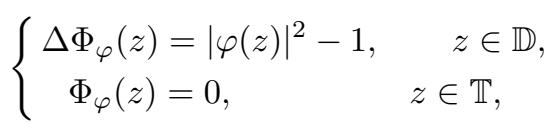

and it is interesting to note that in terms of the function $\Phi_{\varphi}$, the condition that $\varphi$ be an inner divisor may be written in a more explicit form: $\nabla \Phi_{\varphi}=0$ on $\mathbb{T}$ (in a weak sense if $\Phi_{\varphi}$ is not continuously differentiable up to the boundary $\mathbb{T}$ ). Here, $\nabla$ denotes the gradient operator.

The following result was proved in $[33,37]$. It proved to be the starting point for further development. 
THEOREM 2.4 If $\varphi$ is an inner divisor for $L_{a}^{2}(\mathbb{D})$, then the function $\Phi_{\varphi}$ meets

$$
0 \leq \Phi_{\varphi}(z) \leq 1-|z|^{2}, \quad z \in \mathbb{D}
$$

and we have the isometry

$$
\|\varphi f\|_{L^{2}}^{2}=\|f\|_{L^{2}}^{2}+\int_{\mathbb{D}}\left|f^{\prime}(z)\right|^{2} \Phi_{\varphi}(z) d S(z),
$$

valid for all $f \in H^{2}(\mathbb{D})$. As a consequence, we have

$$
\|f\|_{L^{2}} \leq\|\varphi f\|_{L^{2}} \leq\|f\|_{H^{2}}, \quad f \in H^{2}(\mathbb{D}) .
$$

PROBLEM 4 Does the isometry (2.1) in Theorem 2.4 extend to all $f \in L_{a}^{2}(\mathbb{D})$, with the understanding that if one of the sides equals $+\infty$, then so does the other? If not, then for which inner divisors is this so?

It is possible to prove that the isometry (2.1) holds for zero divisors $\varphi$. However, it turns out that the first question of Problem 4 has a negative answer in general; see Borichev's and Hedenmalm 's paper [20] for a (complicated) counterexample.

Theorem 2.4 has the following consequence (see [33, 37]).

COROLLARY 2.5 Let $A$ be a zero sequence for the space $L_{a}^{2}(\mathbb{D})$. Then the zero divisor $\varphi_{A}$ possesses no other zeros in $\mathbb{D}$ than those of the sequence $A$. Furthermore, it has the property that every function $f \in L_{a}^{2}(\mathbb{D})$ that vanishes on $A$ admits a factoring $f=\varphi_{A} g$, with $g \in L_{a}^{2}(\mathbb{D})$, and $\|g\|_{L^{2}} \leq\|f\|_{L^{2}}$.

This means that although the zero divisors are not isometric divisors as in the Hardy space setting, at least they are contractive divisors.

\section{Properties of zero divisors}

It is a consequence of Theorem 2.4 that if $\varphi$ is an inner divisor for $L_{a}^{2}(\mathbb{D})$, we have

$$
\left\|\left.f\right|_{L^{2}} \leq\right\| \varphi f \|_{L^{2}}, \quad f \in H^{2}(\mathbb{D})
$$

which may be written as $1 \prec \varphi$, in the notation introduced by Korenblum [57]. The precise definition of $\varphi \prec \psi$, for $\varphi, H \in L_{a}^{2}(\mathbb{D})$, is

$$
\|\varphi f\|_{L^{2}} \leq\|\psi f\|_{L^{2}}, \quad f \in H^{\infty}(\mathbb{D}) .
$$

The zero divisor for the empty zero sequence is $\varphi_{\emptyset}=1$, so we may interpret the statement $1 \prec \varphi_{A}$ which follows from Corollary 2.5 (or Theorem 2.4) as $\varphi_{\emptyset} \prec \varphi_{A}$. Here, as always, $\varphi_{A}$ is the zero divisor associated with a zero sequence $A$. Maybe $\varphi_{\emptyset} \prec \varphi_{A}$ should be thought of as a consequence of the fact that $\emptyset \subset A$ holds for all $A$ ? This hints that the following may be true.

THEOREM 3.1 (Hedenmalm, Jakobsson, Shimorin) If $A, B$ are two zero sequences for $L_{a}^{2}(\mathbb{D})$ having $A \subset B$, then $\varphi_{A} \prec \varphi_{B}$.

This result was conjectured by Hedenmalm in 1992, and finally proved by Hedenmalm, Jakobsson, and Shimorin in [46, 47] (see also [50]). It turns out that it is related to a certain maximum principle for biharmonic operators on negatively curved surfaces.

We write here $\Phi_{A}$ instead of $\Phi_{\varphi_{A}}$, for a given zero sequence $A$. In view of Theorem 2.4, Theorem 3.1 is a consequence of the following result. 
THEOREM 3.2 (Hedenmalm, Jakobsson, Shimorin) If $A, B$ are two finite zero sequences for $L_{a}^{2}(\mathbb{D})$ having $A \subset B$, then $\Phi_{A} \leq \Phi_{B}$ holds on $\mathbb{D}$.

REMARK 3.3 Once Theorem 3.2 has been obtained, a limit process argument asserts that

$$
\Phi_{A}(z) \leq \Phi_{B}(z), \quad z \in \mathbb{D},
$$

holds for general Bergman zero sequences $A, B$ with $A \subset B$.

The following result connects Theorem 3.2 with Problem 4.

PROPOSITION 3.4 The isometry

$$
\left\|\varphi_{A} f\right\|_{L^{2}}^{2}=\|f\|_{L^{2}}^{2}+\int_{\mathbb{D}}\left|f^{\prime}(z)\right|^{2} \Phi_{A}(z) d S(z),
$$

holds for all holomorphic functions $f$ on $\mathbb{D}$, and all zero sequences $A$ in $\mathbb{D}$ for the space $L_{a}^{2}(\mathbb{D})$.

Proof. We first treat the case when the sequence $A$ is finite. If $f$ is analytic on $\mathbb{D}$, and $r$ has $0<r<1$, consider the dilation $f_{r}$ of $f$,

$$
f_{r}(z)=f(r z), \quad z \in \mathbb{D},
$$

which clearly belongs to the space $H^{2}(\mathbb{D})$. By the isometry of Theorem 1.6, we have

$$
\left\|\varphi_{A} f_{r}\right\|_{L^{2}}^{2}=\left\|f_{r}\right\|_{L^{2}}^{2}+\int_{\mathbb{D}}\left|f_{r}^{\prime}(z)\right|^{2} \Phi_{A}(z) d S(z) .
$$

Since $A$ is finite, we know that $\varphi_{A}$ is bounded away from 0 and $\infty$ in a small neighborhood of the circle $\mathbb{T}$ [33], and by Theorem 2.4, $\Phi_{A} \geq 0$. Therefore, if we let $r$ tend to 1 in the above identity, with the understanding that if one side takes the value $+\infty$, then so does the other, we obtain in the limit

$$
\left\|\varphi_{A} f\right\|_{L^{2}}^{2}=\|f\|_{L^{2}}^{2}+\int_{\mathbb{D}}\left|f^{\prime}(z)\right|^{2} \Phi_{A}(z) d S(z) .
$$

We turn to the remaining case of infinite $A$. We then write $A=\left\{a_{j}\right\}_{j=1}^{\infty}$, and denote by $A_{N}$ the finite subsequence $\left\{a_{j}\right\}_{j=1}^{N}$. By the above argument, we have the isometry

$$
\left\|\varphi_{A_{N}} f\right\|_{L^{2}}^{2}=\|f\|_{L^{2}}^{2}+\int_{\mathbb{D}}\left|f^{\prime}(z)\right|^{2} \Phi_{A_{N}}(z) d S(z), \quad f \in \mathcal{O}(\mathbb{D})
$$

for all positive integers $N$, where $\mathcal{O}(\mathbb{D})$ denotes the Fréchet space of all holomorphic functions on $\mathbb{D}$. We write $g=\varphi_{A} f$, and apply (3.1) to the function $g / \varphi_{A_{N}}$, to get

$$
\|g\|_{L^{2}}^{2}=\left\|g / \varphi_{A_{N}}\right\|_{L^{2}}^{2}+\int_{\mathbb{D}}\left|\left(g / \varphi_{A_{N}}\right)^{\prime}(z)\right|^{2} \Phi_{A_{N}}(z) d S(z) .
$$

Letting $N \rightarrow+\infty$, an application of Fatou's lemma yields

$$
\left\|g / \varphi_{A}\right\|_{L^{2}}^{2}+\int_{\mathbb{D}}\left|\left(g / \varphi_{A}\right)^{\prime}(z)\right|^{2} \Phi_{A}(z) d S(z) \leq\|g\|_{L^{2}}^{2} .
$$

Remembering that $g$ was the function $\varphi_{A} f$, the inequality

$$
\|f\|_{L^{2}}^{2}+\int_{\mathbb{D}}\left|f^{\prime}(z)\right|^{2} \Phi_{A}(z) d S(z) \leq\left\|\varphi_{A} f\right\|_{L^{2}}^{2}
$$


follows.

We now proceed to obtain the claimed isometry. By the above, all we need to do is to obtain the reverse inequality in (3.2). By Theorem 3.2 and the monotone convergence theorem, the right hand side of the identity (3.1) converges to

$$
\|f\|_{L^{2}}^{2}+\int_{\mathbb{D}}\left|f^{\prime}(z)\right|^{2} \Phi_{A}(z) d S(z)
$$

as $N \rightarrow+\infty$, and by Fatou's lemma,

$$
\left\|\varphi_{A} f\right\|_{L^{2}} \leq \limsup _{N \rightarrow+\infty}\left\|\varphi_{A_{N}} f\right\|_{L^{2}}, \quad f \in \mathcal{O}(\mathbb{D}) .
$$

We conclude that

$$
\left\|\varphi_{A} f\right\|_{L^{2}}^{2} \leq\|f\|_{L^{2}}^{2}+\int_{\mathbb{D}}\left|f^{\prime}(z)\right|^{2} \Phi_{A}(z) d S(z), \quad f \in \mathcal{O}(\mathbb{D}) .
$$

The proof of Proposition 3.4 is complete.

REMARK 3.5 If we denote by $\mathcal{H}(A)$ the Hilbert space of holomorphic functions in $\mathbb{D}$ with norm

$$
\|f\|_{\mathcal{H}(A)}^{2}=\|f\|_{L^{2}}^{2}+\int_{\mathbb{D}}\left|f^{\prime}(z)\right|^{2} \Phi_{A}(z) d S(z),
$$

we may reformulate the assertion of Proposition 3.4 as follows: if $f$ is holomorphic in $\mathbb{D}$, and $A$ is a zero sequence for $L_{a}^{2}(\mathbb{D})$, then $\varphi_{A} f \in L_{a}^{2}(\mathbb{D})$ if and only if $f \in \mathcal{H}(A)$, and

$$
\|f\|_{\mathcal{H}(A)}=\left\|\varphi_{A} f\right\|_{L^{2}} .
$$

\section{Connections with potential theory and partial dif- ferential equations}

It was Peter Duren, Dmitry Khavinson, Harold Shapiro, and Carl Sundberg who found the connection between the potential function $\Phi_{\varphi}$ for a given inner divisor and the biharmonic Green function for the disk $\mathbb{D}$. The biharmonic Green function is defined by the expression

$$
\Gamma(z, \zeta)=|z-\zeta|^{2} G(z, \zeta)+\left(1-|z|^{2}\right)\left(1-|\zeta|^{2}\right), \quad(z, \zeta) \in \mathbb{D}^{2},
$$

and a calculation shows that it is positive on the bidisk $\mathbb{D} \times \mathbb{D}$. Here, $G(z, \zeta)$ is the usual Green function for $\Delta$ :

$$
G(z, \zeta)=\log \left|\frac{\zeta-z}{1-\bar{\zeta} z}\right|^{2}, \quad(z, \zeta) \in \mathbb{D}^{2}
$$

The biharmonic Green function solves the PDE boundary value problem

$$
\left\{\begin{array}{lc}
\Delta_{z}^{2} \Gamma(z, \zeta)=\delta_{\zeta}(z), & z \in \mathbb{D}, \\
\Gamma(z, \zeta)=0, & z \in \mathbb{T}, \\
\nabla_{z} \Gamma(z, \zeta)=0, & z \in \mathbb{T},
\end{array}\right.
$$

for a given interior point $\zeta \in \mathbb{D}$. The reason is basically that an application of Green's formula yields the representation

$$
\Phi_{\varphi}(z)=\int_{\mathbb{D}} \Gamma(z, \zeta) \Delta_{\zeta}\left(|\varphi(\zeta)|^{2}-1\right) d S(\zeta)=\int_{\mathbb{D}} \Gamma(z, \zeta)\left|\varphi^{\prime}(\zeta)\right|^{2} d S(\zeta) \geq 0 .
$$


The way to obtain Theorem 3.2 (and hence Theorem 3.1 as well) in a similar manner would be to take advantage the positivity of a similar weighted biharmonic operator on $\mathbb{D}$, which corresponds to the bilaplacian on a certain Riemann surface which we map to the unit disk via conformal mapping. The branch points for the Riemann surface correspond to the zeros of the finite zero divisor $\varphi$.

THEOREM 4.1 (Hedenmalm, Jakobsson, Shimorin) The Green function for the singular fourth order elliptic operator $\Delta|\varphi|^{-2} \Delta$ is positive on $\mathbb{D} \times \mathbb{D}$ for every finite zero divisor $\varphi$ for the space $L_{a}^{2}(\mathbb{D})$. Here, we mean by the Green function the solution $\Gamma_{|\varphi|^{2}}(z, \zeta)$ to the problem

$$
\left\{\begin{array}{lc}
\Delta_{z}|\varphi(z)|^{-2} \Delta_{z} \Gamma_{|\varphi|^{2}}(z, \zeta)=\delta_{\zeta}(z), & z \in \mathbb{D} \\
\Gamma_{|\varphi|^{2}}(z, \zeta)=0, & z \in \mathbb{T} \\
\nabla_{z} \Gamma_{|\varphi|^{2}}(z, \zeta)=0, & z \in \mathbb{T}
\end{array}\right.
$$

We supply the argument connecting Theorems 3.2 and 4.1. Let $A$ and $B$ be two finite sequences of points in the disk, having $A \subset B$. The difference function $\Phi_{B, A}=\Phi_{B}-\Phi_{A}$ solves the overdetermined problem

$$
\left\{\begin{array}{cc}
\Delta \Phi_{B, A}(z)=\left|\varphi_{B}(z)\right|^{2}-\left|\varphi_{A}(z)\right|^{2}, & z \in \mathbb{D} \\
\Phi_{B, A}(z)=0, & z \in \mathbb{T}, \\
\nabla \Phi_{B, A}(z)=0, & z \in \mathbb{T}
\end{array}\right.
$$

and as we divide both sides of the top line by $\left|\varphi_{A}(z)\right|^{2}$, we get

$$
\left|\varphi_{A}(z)\right|^{-2} \Delta \Phi_{B, A}(z)=\left|\varphi_{B}(z) / \varphi_{A}(z)\right|^{2}-1, \quad z \in \mathbb{D} .
$$

Since we have overdetermined boundary values, we are at liberty to apply another Laplacian, which results in

$$
\begin{cases}\Delta\left|\varphi_{A}(z)\right|^{-2} \Delta \Phi_{B, A}(z)=\left|\left(\frac{\varphi_{B}}{\varphi_{A}}\right)^{\prime}(z)\right|^{2}, & z \in \mathbb{D} \\ \Phi(z)=0, & z \in \mathbb{T} \\ \nabla \Phi(z)=0, & z \in \mathbb{T}\end{cases}
$$

Note here that we used the fact that the quotient $\varphi_{B} / \varphi_{A}$ is holomorphic on $\mathbb{D}$. Finally, we see that in terms of the weighted biharmonic Green function $\Gamma_{\left|\varphi_{A}\right|^{2}}(z, \zeta)$, we may express $\Phi_{B, A}$ as

$$
\Phi_{B, A}(z)=\int_{D} \Gamma_{\left|\varphi_{A}\right|^{2}}(z, \zeta)\left|\left(\frac{\varphi_{B}}{\varphi_{A}}\right)^{\prime}(\zeta)\right|^{2} d S(\zeta), \quad z \in \mathbb{D},
$$

and the positivity of $\Phi_{B, A}$ is now immediate.

The connection between this weighted situation and general hyperbolic geometry is explained extensively in [47].

The first results suggesting the validity of Theorem 4.1 were calculations made in [38] and [39], dealing with a multiple zero at the origin and a single zero at an arbitrary point of $\mathbb{D}$, respectively. Then a multiple zero arbitrarily located in the disk was considered, based on the computations of Hansbo [30]. This convinced us the claim must be true, but it took a lot of additional effort to carry out the proof, which involves the construction of a Hele-Shaw flow [50], to be able to take advantage of an additional invariance property. We recall that the finite zero divisor $\varphi$ has the property

$$
h(0)=\int_{\mathbb{D}} h(z)|\varphi(z)|^{2} d S(z)
$$


for all bounded harmonic functions $h$ on $\mathbb{D}$. Now, let $0<t<1$, and suppose $D(t)$ is a subdomain of $\mathbb{D}$ containing the origin for which

$$
t h(0)=\int_{D(t)} h(z)|\varphi(z)|^{2} d S(z)
$$

holds for all bounded harmonic functions $h$ on $D(t)$; note that in the limit, we should get $D(1)=\mathbb{D}$. It turns out that this property uniquely determines the domain $D(t)$, and that these domains increase with the parameter $t$ in a predictable fashion (at least this was proved in a slightly smoother situation in [50]). This offers the opportunity to vary $t$, which is done quite successfully in [47]. An important question remained.

CONJECTURE 4.2 For a finite zero divisor $\varphi$, the weighted biharmonic Green function has the property

$$
\frac{\partial}{\partial n(z)} \Delta_{z} \Gamma_{|\varphi|^{2}}(z, \zeta) \geq 0, \quad(z, \zeta) \in \mathbb{T} \times \mathbb{D}
$$

the normal derivative being taken in the exterior direction.

It turns out that if this claim could be verified, then the following quite pleasant property of the functions $\varphi_{B} / \varphi_{A}$ would follow.

CONJECTURE 4.3 Let $A, B$ be two finite zero divisors, with $A \subset B$. Then the argument of $\varphi_{B}(z) / \varphi_{A}(z)$ increases monotonically as $z$ is moved counterclockwise along the unit circle $\mathbb{T}$. In particular, if $B \backslash A$ is a single point, then $\varphi_{B} / \varphi_{A}$ is star-shaped univalent function on $\mathbb{D}$.

We turn to a possible generalization of Theorem 4.1 in a different direction. After all, it would be valuable to be able have the entire factorization theory of $L_{a}^{2}(\mathbb{D})$ transferred to the weighted Bergman spaces $L_{a}^{2}\left(\mathbb{D}, \omega_{\alpha}\right)$, defined as consisting of analytic functions $f$ in $\mathbb{D}$ with

where the weight is

$$
\|f\|_{L^{2}\left(\omega_{\alpha}\right)}=\left(\int_{\mathbb{D}}|f(z)|^{2} \omega_{\alpha}(z) d S(z)\right)^{1 / 2},
$$

$$
\omega_{\alpha}(z)=(\alpha+1)\left(1-|z|^{2}\right)^{\alpha},
$$

and $-1<\alpha<+\infty$. It follows from the results in [47] that we have the same factorization theory in these spaces also with respect to Korenblum domination as long as $-1<\alpha \leq 0$. Moreover, it is known from the counterexample of Hedenmalm and Zhu that this is not so for $1<\alpha<+\infty$. There is abundant evidence suggesting that on the remaining interval we also have the same factorization theory involving Korenblum domination (but not going so far as Conjecture 4.3, though), but so far this has not been substantiated. The following is what we need to conclude the issue.

CONJECTURE 4.4 Let $\omega$ be a positive $\mathbb{C}^{\infty}$-smooth weight in $\overline{\mathbb{D}}$, with the reproducing property

$$
h(0)=\int_{\mathbb{D}} h(z) \omega(z) d S(z),
$$

for all bounded harmonic functions $h$ in $\mathbb{D}$. Suppose

$$
\Delta \log \omega(z) \geq \Delta \log \left(1-|z|^{2}\right)=-\frac{1}{\left(1-|z|^{2}\right)^{2}}, \quad z \in \mathbb{D} .
$$

Then the Green function $\Gamma_{\omega}(z, \zeta)$ for the weighted biharmonic operator $\Delta \omega^{-1} \Delta$ is positive on $\mathbb{D} \times \mathbb{D}$. 
In support of the conjecture, we only mention that it is valid for the weight

$$
\omega(z)=2\left(1-|z|^{2}\right), \quad z \in \mathbb{D} .
$$

PROPOSITION 4.5 The Green function $\Gamma^{1}(z, \zeta)$ for the weighted biharmonic operator $\Delta\left(1-|z|^{2}\right)^{-1} \Delta$ has the explicit form

$$
\begin{aligned}
\Gamma^{1}(z, \zeta)= & \left(|z-\zeta|^{2}-\frac{1}{4}\left|z^{2}-\zeta^{2}\right|^{2}\right) G(z, \zeta)+\frac{1}{8}\left(1-|z|^{2}\right)\left(1-|\zeta|^{2}\right) \\
& \times\left(7-|z|^{2}-|\zeta|^{2}-|\zeta z|^{2}-4 \operatorname{Re}(\bar{\zeta} z)-2\left(1-|z|^{2}\right)\left(1-|\zeta|^{2}\right) \operatorname{Re} \frac{1+\bar{\zeta} z}{1-\bar{\zeta} z}\right)
\end{aligned}
$$

for $(z, \zeta) \in \mathbb{D}^{2}$.

It is quickly verified that $\Gamma^{1}>0$ on $\mathbb{D}^{2}$. However, for fixed $\zeta \in \mathbb{D}$, the function $\Gamma^{1}(\cdot, \zeta)$ is very flat at the boundary $\mathbb{T}$; it satisfies

$$
\Gamma^{1}(z, \zeta)=O\left((1-|z|)^{3}\right) \quad \text { as } \quad|z| \rightarrow 1
$$

\section{Generators of zero-based subspaces}

As before, we write $\varphi_{A}$ for the zero divisor associated with a zero sequence $A$.

PROBLEM 5 Does $\varphi_{\text {A }}$ generate

$$
\mathcal{I}(A)=\left\{f \in L_{a}^{2}(\mathbb{D}): f=0 \text { on } A\right\}
$$

as an invariant subspace? In other words, do the functions $\varphi_{A}, z \varphi_{A}, z^{2} \varphi_{A}, \ldots$ span a dense subspace of $\mathcal{I}(A)$ ?

This problem was solved by Aleman, Richter, and Sundberg in [10]. The main tool was a dilation property of the biharmonic Green function $\Gamma$, which showed that

$$
\varphi_{A} f_{r} \rightarrow \varphi f \quad \text { as } \quad r \rightarrow 1^{-}
$$

in the norm of $L_{a}^{2}(\mathbb{D})$, for any $f \in L_{a}^{2}(\mathbb{D})$ with $\varphi_{A} f \in L_{a}^{2}(\mathbb{D})$ as well. Here, $f_{r}(z)=f(r z)$ is the dilation of $f$. This way to do it is essentially the approach that is suggested by Problem 7 and Remark 5.2 (see below). Another way to solve Problem 5 is to try to apply Theorem 4.1, which leads to the following question.

PROBLEM 6 Is the function $\varphi_{A \cup\{0\}} / \varphi_{A}$ always bounded in the disk $\mathbb{D}$ ?

This problem was solved in the affirmative by Shimorin [75]; Hedenmalm later modified the approach to obtain a sharp off-diagonal estimate of weighted Bergman kernels, with logarithmically subharmonic weights that reproduce for the origin. Actually, the function

$$
\left|\frac{\varphi_{A \cup\{\alpha\}}(z)}{\varphi_{A}(z)}\right| \leq 1+\frac{1-|\alpha|^{2}}{|1-\bar{\alpha} z|} \leq 3
$$

for each $\alpha \in \mathbb{D}$, as was shown by Aleman and Richter [8]. Note that this gives the bound 2 for $\alpha=0$. A constant bound that depends on $\alpha$ is supplied by

$$
\left|\frac{\varphi_{A \cup\{\alpha\}}(z)}{\varphi_{A}(z)}\right| \leq \frac{2+|\alpha|}{\sqrt{2-|\alpha|^{2}}} \leq 3,
$$


which also follows from the work in [8]. This gives the optimal bound $\sqrt{2}$ for $\alpha=0$. See also Section 12 for further details on the methods.

By Theorem 4.1, we have $\varphi_{A} \prec \varphi_{A \cup\{0\}}$, which entails that

$$
\left|z \varphi_{A}(z)\right| \leq\left|\varphi_{A \cup\{0\}}(z)\right|, \quad z \in \mathbb{D},
$$

holds. To see this, check it for finite sequences $A$ (the argument for this is analogous to what was used in Proposition 1.3 [33]), and we then approximate a general zero sequence with finite subsequences. It follows that $\varphi_{A \cup\{0\}} / \varphi_{A}$ belongs to the Nevanlinna class of holomorphic quotients of bounded analytic functions. But, by Shimorin's result, we know it is bounded itself.

REMARK 5.1 We should shed some light on the connection between Problems 5 and 6. To do this, we assume for simplicity that the point 0 does not belong to the given zero sequence $A$, and denote by $Y_{n}$ the orthogonal projection onto $\mathcal{I}\left(A \cup O_{n}\right)$ of the function $z^{n}$. Here, $O_{n}$ stands for the the sequence that consists of $n$ copies of the point 0 . The assumption that 0 not belong to $A$ prevents $Y_{n}$ from collapsing to 0 . We now claim that the functions $Y_{n}, n=0,1,2, \ldots$, span a dense subspace of $\mathcal{I}(A)$. To this end, suppose $f \in \mathcal{I}(A)$ is orthogonal to all the functions $Y_{n}$. It is convenient here to introduce $X_{n}=z^{n}-Y_{n}$, which for each $n$ is orthogonal to $\mathcal{I}\left(A \cup O_{n}\right)$, by the way we defined the element $Y_{n}$. If we knew $f$ belonged to $\mathcal{I}\left(A \cup O_{n}\right)$ for some particular $n$, we would then also have $\left\langle f, X_{n}\right\rangle_{L^{2}}=0$, and since by assumption $\left\langle f, Y_{n}\right\rangle_{L^{2}}=0$, we see that

$$
\frac{f^{(n)}(0)}{(n+1) !}=\left\langle f, z^{n}\right\rangle_{L^{2}}=0 .
$$

We conclude that $f$ must also belong to $\mathcal{I}\left(A \cup O_{n+1}\right)$. The initial assumption $f \in \mathcal{I}\left(A \cup O_{n}\right)$ is fulfilled for $n=0$, so by induction, $f$ belongs to the intersection of all the spaces $\mathcal{I}\left(A \cup O_{n}\right)$, which is $\{0\}$. This shows that $f=0$, and hence the claim is verified. It is known [33, 23] that the zero divisor for $A \cup O_{n}$ is

$$
\varphi_{A \cup O_{n}}=Y_{n} /\left\|Y_{n}\right\|_{L^{2}}
$$

so by the above argument, the functions $\varphi_{A \cup O_{n}}, n=0,1,2, \ldots$, span a dense subspace of $\mathcal{I}(A)$. If we could only demonstrate that every $\varphi_{A \cup O_{n}}$ belongs to the invariant subspace generated by $\varphi_{A}$, this would provide an alternative route toward obtaining an affirmative solution to Problem 5. This is where it is good that Problem 6 has an affirmative solution as well. For, we then know that $\varphi_{A \cup O_{n+1}} / \varphi_{A \cup O_{n}}$ is bounded for each $n=0,1,2, \ldots$, so that the function $\varphi_{A \cup O_{n}} / \varphi_{A}$ is bounded, too, for each $n=0,1,2, \ldots$ It is now immediate that $\varphi_{A \cup O_{n}}$ belongs to the invariant subspace generated by $\varphi_{A}$, and hence $\varphi_{A}$ generates all of $\mathcal{I}(A)$ as an invariant subspace.

We really do not understand the process of adding another zero. Nevertheless, for the function $\lambda_{A}(z)=\bar{\varphi}_{A}(0) \varphi_{A}(z)$, there is the iterative formula

$$
\lambda_{A \cup\{\beta\}}(z)=L_{A}(z)-\frac{\lambda_{A}(\beta)}{\lambda_{\varphi_{\beta}(A)}(0)} \varphi_{\beta}^{\prime}(z) \lambda_{\varphi_{\beta}(A)}\left(\varphi_{\beta}(z)\right),
$$

where $\varphi_{\beta}$ denotes the Möbius mapping

$$
\varphi_{\beta}(z)=\frac{\beta-z}{1-\bar{\beta} z}, \quad z \in \mathbb{D} .
$$

The starting point for the iterative process is $\lambda_{\emptyset}=1$, and the formula connecting $\varphi_{A}$ with $\lambda_{A}$ may be written

$$
\varphi_{A}(z)=\frac{\lambda_{A}(z)}{\sqrt{L_{A}(0)}}, \quad z \in \mathbb{D} .
$$

The next problem is also related to Problem 5. 
PROBLEM 7 Suppose $\omega$ is a continuous function on $\mathbb{D}$ which satisfies

$$
0 \leq \omega(z) \leq C\left(1-|z|^{2}\right), \quad z \in \mathbb{D},
$$

for some positive constant $C$, which is super-biharmonic:

$$
\Delta^{2} \omega(z) \geq 0, \quad z \in \mathbb{D} .
$$

Must then the polynomials be dense in the weighted Bergman space $L_{a}^{2}(\mathbb{D}, \omega)$ of all holomorphic functions in $\mathbb{D}$ with

$$
\int_{\mathbb{D}}|f(z)|^{2} \omega(z) d S(z)<+\infty ?
$$

REMARK 5.2 We shall now try to indicate the relationship between Problems 5 and 7. Note first that in view of Remark 3.5, $\varphi_{A}$ generates $\mathcal{I}(A)$ if and only if the closure of polynomials is dense in the space $\mathcal{H}(A)$ (defined in the indicated remark). By the elementary estimates

$$
\frac{2}{3}\|f\|_{L^{2}}^{2} \leq \int_{\mathbb{D}}\left|f^{\prime}(z)\right|^{2}\left(1-|z|^{2}\right)^{2} d S(z) \leq 2\|f\|_{L^{2}}^{2},
$$

valid for $f \in L_{a}^{2}(\mathbb{D})$ with $f(0)=0$, we have that the norm in $\mathcal{H}(A)$ is comparable to

$$
\|f\|_{*}^{2}=|f(0)|^{2}+\int_{\mathbb{D}}\left|f^{\prime}(z)\right|^{2} \omega_{A}(z) d S(z),
$$

where we denote by $\omega_{A}$ the function

$$
\omega_{A}(z)=\left(1-|z|^{2}\right)^{2}+\Phi_{A}(z), \quad z \in \mathbb{D} .
$$

We see that the polynomials are dense in $\mathcal{H}(A)$ if and only if they are dense in $L_{a}^{2}\left(\mathbb{D}, \omega_{A}\right)$. It is now clear that $\varphi_{A}$ generates the invariant subspace $\mathcal{I}(A)$ if and only if approximation by the polynomials is possible in $L_{a}^{2}\left(\mathbb{D}, \omega_{A}\right)$. The constructed function $\omega_{A}$ has

$$
0 \leq\left(1-|z|^{2}\right)^{2} \leq \omega_{A}(z) \leq\left(1-|z|^{2}\right)^{2}+1-|z|^{2} \leq 2\left(1-|z|^{2}\right), \quad z \in \mathbb{D},
$$

by Theorem 2.4. Moreover, since $\Delta^{2} \Phi_{A} \geq 0$, we also have

$$
\Delta \omega_{A}(z)=\Delta^{2} \Phi_{A}(z)+\Delta^{2}\left(1-|z|^{2}\right)^{2} \geq \Delta^{2}\left(1-|z|^{2}\right)^{2}=4 \geq 0, \quad z \in \mathbb{D} .
$$

Polynomial approximation problems are, generally speaking, rather difficult. It turns out that the Problem 7 as stated above has an affirmative answer, and the reason is that the biharmonic Green function has certain "good" dilation properties (see [3] and [2]; the problem is actually partially solved already in [10]).

Proposition 5.3 below represents our current level of understanding on the general topic of weighted polynomial approximation. Given a positive continuous weight function $\omega$ on the unit disk $\mathbb{D}$, having

$$
\int_{\mathbb{D}} \omega(z) d S(z)<+\infty
$$

we denote by $L_{h}^{2}(\mathbb{D}, \omega)$ the Hilbert space of harmonic functions $f$ on $\mathbb{D}$ having

$$
\|f\|_{L^{2}(\omega)}=\left(\int_{\mathbb{D}}|f(z)|^{2} \omega(z) d S(z)\right)^{1 / 2}<+\infty .
$$


It has been known for a long time [61, p. 131], [54, p. 343], that the analytic polynomials are dense in $L_{a}^{2}(\mathbb{D}, \omega)$ for radial weights $\omega$. The corresponding statement is also true for the space $L_{h}^{2}(\mathbb{D}, \omega)$, and moreover, we can get the result for weights that do not deviate too much from radial weights. To obtain such a result, it is useful to consider for a parameter $0<\lambda<1$ and a function $f \in L_{h}^{2}(\mathbb{D}, \omega)$ the dilation $f_{\lambda}$ of $f$ :

$$
f_{\lambda}(z)=f(\lambda z), \quad z \in \mathbb{D},
$$

and observe that every dilation $f_{\lambda}$ of $f$ is definitely approximable by harmonic polynomials (or analytic polynomials, if $f \in L_{a}^{2}(\mathbb{D}, \omega)$ ), so that if we could show that $f_{\lambda} \rightarrow f$ in the norm of $L^{2}(\mathbb{D}, \omega)$, the desired conclusion would follow. Another condition which is known to assure that we have polynomial approximation is due to Dzhrbashian [61, p.133], and requires that the weight should (almost) fall on every radius emanating from the origin. If we merge these two ideas, we obtain the following result. First, however, we need to recall some terminology: an integrable function $\nu \geq 0$ on the unit circle $\mathbb{T}$ meets the Muckenhoupt $\left(A_{2}\right)$ condition provided that

$$
\mathfrak{A}_{2}(\nu)=\sup _{I}\left\{|I|^{-2} \int_{I} \nu d s \int_{I} \frac{1}{\nu} d s\right\}<\infty,
$$

the supremum being taken over all $\operatorname{arcs} I$ on $\mathbb{T}$, where $d s$ denotes arc length measure on $\mathbb{T}$, normalized so that the total length of $\mathbb{T}$ is 1 .

PROPOSITION 5.3 Suppose $\omega$ is a positive continuous function on the unit disk $\mathbb{D}$, which meets the integrability condition (5.1). Suppose, moreover, that $\left\{\lambda_{j}\right\}_{1}^{\infty}$ is a sequence of numbers in the interval ]0,1[, converging to 1 . For $r, 0<r<1$, let $\omega_{r}(z)=\omega(r z)$, and for $0<r, s<1$, introduce the quantity

$$
\mathfrak{Q}[\omega](r, s)=\sup \{\omega(r z) / \omega(s z): z \in \mathbb{T}\} .
$$

If the weight $\omega$ satisfies

$$
\limsup _{r \rightarrow 1-} \sup _{j}\left(\min \left\{\mathfrak{Q}[\omega]\left(r, \lambda_{j} r\right), \mathfrak{A}_{2}\left(\omega_{r}\right)\right\}\right)<\infty,
$$

then the dilations $f_{\lambda_{j}}$ of $f$ converge to $f$ as $j \rightarrow+\infty$ in the norm of $L_{h}^{2}(\mathbb{D}, \omega)$, for every $f \in L_{h}^{2}(\mathbb{D}, \omega)$. As a consequence, under this condition on $\omega$, we see that the harmonic polynomials are dense in $L_{h}^{2}(\mathbb{D}, \omega)$, and the analytic polynomials are dense in $L_{a}^{2}(\mathbb{D}, \omega)$.

Proof. We follow the general line of argument of [54, pp. 343-344]. Given an $\varepsilon$, $0<\varepsilon$, take $\rho, 0<\rho<1$, so close to 1 that

$$
\int_{\rho<|z|<1}|f(z)|^{2} \omega(z) d S(z)<\varepsilon
$$

and

$$
\int_{\lambda_{j} \rho<|z|<\lambda_{j}}|f(z)|^{2} \omega(z) d S(z)<\lambda_{j}^{2} \varepsilon .
$$

By choosing $\rho$ possibly even closer to 1 , we may assume that

$$
\min \left\{\mathfrak{Q}[\omega]\left(r, \lambda_{j} r\right), \mathfrak{A}_{2}\left(\omega_{r}\right)\right\} \leq C, \quad \rho<r<1, j=1,2,3, \ldots,
$$

for some constant $C, 0<C<+\infty$. We now plan to estimate the size of

$$
\int_{\rho<|z|<1}\left|f\left(\lambda_{j} z\right)\right|^{2} \omega(z) d S(z)
$$


We fix an $r, \rho<r<1$, and note that if it is $\mathfrak{Q}[\omega]\left(r, \lambda_{j} r\right)$ that is $\leq C$, we have

$$
\omega\left(r e^{i \theta}\right) \leq C \omega\left(\lambda_{j} r e^{i \theta}\right)
$$

and hence

$$
\int_{-\pi}^{\pi}\left|f\left(\lambda_{j} r e^{i \theta}\right)\right|^{2} \omega\left(r e^{i \theta}\right) d \theta \leq C \int_{-\pi}^{\pi}\left|f\left(\lambda_{j} r e^{i \theta}\right)\right|^{2} \omega\left(\lambda_{j} r e^{i \theta}\right) d \theta .
$$

If, on the other hand, it is $\mathfrak{A}_{2}\left(\omega_{r}\right)$ that is $\leq C$, then by Muckenhoupt's theorem [62, p. 223], (and the control of the constants involved [62, pp. 215, 224], we have

$$
\int_{-\pi}^{\pi}\left|f\left(\lambda_{j} r e^{i \theta}\right)\right|^{2} \omega\left(r e^{i \theta}\right) d \theta \leq K(C) \int_{-\pi}^{\pi}\left|f\left(r e^{i \theta}\right)\right|^{2} \omega\left(r e^{i \theta}\right) d \theta
$$

for some constant $K(C)$ that only depends on $C$. No matter which is the case, we get

$$
\int_{\rho} 1 \int_{-\pi}^{\pi}\left|f\left(\lambda_{j} r e^{i \theta}\right)\right|^{2} \omega\left(r e^{i \theta}\right) d \theta r d r \leq(C+K(C)) \pi \varepsilon
$$

in view of (5.2) and (5.3). Since $\rho, 0<\rho<1$, was fixed, we have that $f_{\lambda_{j}} \rightarrow f$ as $j \rightarrow+\infty$ uniformly on the disk $|z|<\rho$, and in particular, we can arrange so that

$$
\int_{|z|<\rho}\left|f\left(\lambda_{j} z\right)-f(z)\right|^{2} \omega(z) d S(z)<\varepsilon
$$

for all large $j$, say $j \geq N(\varepsilon)$. If we combine this with the estimate of the integral on the annulus $\rho<|z|<1$, we see that

$$
\int_{\mathbb{D}}\left|f\left(\lambda_{j} z\right)-f(z)\right|^{2} \omega(z) d S(z)<8(1+C+K(C)) \varepsilon,
$$

for $j \geq N(\varepsilon)$. The assertion of the proposition is now immediate.

\section{A Carathéodory theorem for the Bergman space}

Recall the statement of the famous Carathéodory theorem.

THEOREM 6.1 (Carathéodory) Every $f \in H^{\infty}(\mathbb{D})$ with norm $\leq 1$ is the normal limit of finite Blaschke products.

The appropriate analog in a Bergman space setting turns out to be as follows.

CONJECTURE 6.2 Suppose $f$ is analytic on $\mathbb{D}$ with the property

$$
\int_{\mathbb{D}} h(z)|f(z)|^{2} d S(z) \leq h(0)
$$

for all positive and bounded harmonic functions $h$ in $\mathbb{D}$. Then $f$ is the normal limit of finite zero divisors.

In the previous version of this text [39], the formulation of this conjecture was slightly different, in terms of contractive multiplicativity from $H^{2}(\mathbb{D})$ into $L_{a}^{2}(\mathbb{D})$. That formulation has not yet found an answer, and it is quite likely that the answer is negative. In hindsight, the above bersion seems much more appropriate. And it was settled affirmatively by Shimorin in [75]. The proof can also be found in the book [45, Ch. 3]. 


\section{A Frostman theorem for the Bergman space?}

We recall Frostman's classical theorem on approximation of inner functions by Blaschke products.

THEOREM 7.1 (Frostman) Every inner function is approximable in the norm of the space $H^{\infty}(\mathbb{D})$ by Blaschke products.

Let $\mathfrak{M}\left(H^{2}, L_{a}^{2}\right)$ be the space of multipliers $H^{2}(\mathbb{D}) \rightarrow L_{a}^{2}(\mathbb{D})$, normed appropriately:

$$
\|\varphi\|_{\mathfrak{M}\left(H^{2}, L_{a}^{2}\right)}=\sup \left\{\|\varphi f\|_{L^{2}}: f \in H^{2}(\mathbb{D}),\|f\|_{H^{2}} \leq 1\right\} .
$$

Note that in view of Theorem 2.4, every inner divisor is a contractive multiplier $H^{2}(\mathbb{D}) \rightarrow$ $L_{a}^{2}(\mathbb{D})$. This suggests that the above multiplier norm might be the appropriate replacement of the norm in $H^{\infty}(\mathbb{D})$ in the Bergman space setting. The approriate analogue of Frostman's theorem might therefore be the result of asking the following question.

PROBLEM 8 Is every inner divisor for $L_{a}^{2}(\mathbb{D})$ is approximable by zero divisors in the norm of $\mathfrak{M}\left(H^{2}, L_{a}^{2}\right)$ ?

So far, there appears to be no progress on this problem.

\section{Korenblum's maximum principle}

CONJECTURE 8.1 (Korenblum) There exists an absolute constant $\varepsilon, 0<\varepsilon<2^{-1 / 2}$, such that if $f, g \in L_{a}^{2}(\mathbb{D})$ have $|f(z)| \leq|g(z)|$ in the annulus $\varepsilon<|z|<1$, then $\|f\|_{L^{2}} \leq$ $\|g\|_{L^{2}}$.

One can rather trivially obtain an estimate like $\|f\|_{L^{2}} \leq C(\varepsilon)\|g\|_{L^{2}}$, with $C(\varepsilon)$ being a constant larger than 1 tending to 1 as $\varepsilon \rightarrow 0$. Korenblum claims that $C(\varepsilon)=1$ is attained for some nonzero value of the parameter $\varepsilon$. One should view Conjecture 8.1 as a suspected property peculiar to square moduli of analytic functions. If one should try to replace this class by, for instance, the collection of exponentials of subharmonic functions, the analogous assertion that $\varphi(z) \leq \psi(z)$ on the annulus $\varepsilon<|z|<1$ should imply

$$
\int_{\mathbb{D}} \exp (\varphi(z)) d S(z) \leq \int_{\mathbb{D}} \exp (\psi(z)) d S(z)
$$

for subharmonic functions $\varphi, \psi$ fails, no matter how small the positive number $\varepsilon$ is. This is so because one can take as $\varphi(z)$ the function $\log |z|$, and as $\psi(z)$ the function that is the maximum of $\log |z|$ and the constant function $\log \varepsilon$.

The condition of Conjecture 8.1 is invariant under multiplication by a bounded holomorphic function, so the assertion of Conjecture 8.1 may be rephrased as $f \prec g$.

Conjecture 8.1 was settled in 1998 by Hayman [32], with a proof that uses surprisingly simple ingredients. He was helped by Hinkkanen to improve his inner radius to $\varepsilon=\frac{1}{25}$.

The properties of the domination relation $\prec$ deserve to be studied in some depth.

PROBLEM 9 Suppose $f, g \in L_{a}^{2}(\mathbb{D})$ have $f \prec g$ and $g \prec f$. Must then $f=\gamma g$ for $a$ unimodular constant $\gamma$ ?

It turns out that Stefan Richter [64] solved this problem in the affirmative already back in 1988. We outline the argument briefly. Consider the following transform of $|f|^{2}-|g|^{2}$ :

$$
B\left[|f|^{2}-|g|^{2}\right](\lambda, \mu)=(1-\lambda \bar{\mu})^{2} \int_{\mathbb{D}} \frac{|f(z)|^{2}-|g(z)|^{2}}{(1-\lambda \bar{z})^{2}(1-\bar{\mu} z)^{2}} d S(z), \quad(\lambda, \mu) \in \mathbb{D}^{2} .
$$


We know that this function is holomorphic in $\lambda$ and anti-holomorphic in $\mu$. Moreover, $f \prec g$ and $g \prec f$ together force the equality

$$
B\left[|f|^{2}-|g|^{2}\right](\lambda, \lambda)=0, \quad \lambda \in \mathbb{D} .
$$

This means that the holomorphic function of two variables

$$
B\left[|f|^{2}-|g|^{2}\right](\lambda, \bar{\mu})
$$

vanishes along the antidiagonal, which is only possible if the function vanishes throughout $\mathbb{D}^{2}$. But then $|f|^{2}=|g|^{2}$ follows, and the conclusion $f=\gamma g$, with $|\gamma|=1$, is immediate. The above function is related to the so-called Berezin transform [45, Ch. 2].

\section{Cyclic vectors and Shapiro's problem}

The space $A^{-\infty}$ consists of all analytic functions $f$ on the unit disk $\mathbb{D}$ satisfying the growth condition

$$
|f(z)| \leq C(f, \alpha)\left(1-|z|^{2}\right)^{-\alpha}, \quad z \in \mathbb{D},
$$

for some positive constants $\alpha$ and $C(f, \alpha)$. The function theory aspects of this space were illuminated extensively by Korenblum in his Acta paper [55]; one rather trivial but interesting observation is that $A^{-\infty}$ is a topological algebra with respect to pointwise multiplication and the natural injective limit topology. In his second Acta paper [56], Korenblum describes completely the closed ideals in $A^{-\infty}$. The Bergman space $L_{a}^{2}(\mathbb{D})$ is clearly a subspace of $A^{-\infty}$, but it is not an algebra. The invariant subspaces are the $L_{a}^{2}(\mathbb{D})$ analogs of the closed ideals in $A^{-\infty}$. In order to gain some understanding of invariant subspaces, the concept of a cyclic vector is basic.

DEFINITION 9.1 A function $f \in L_{a}^{2}(\mathbb{D})$ is cyclic in $L_{a}^{2}(\mathbb{D})$ if the functions $f, z f$, $z^{2} f, \ldots$ span a dense subspace of $L_{a}^{2}(\mathbb{D})$.

PROBLEM 10 Describe the cyclic elements of $L_{a}^{2}(\mathbb{D})$.

A natural question when one tries to attack Problem 10 is the following.

PROBLEM 11 (Korenblum) It is known that every cyclic element of $L_{a}^{2}(\mathbb{D})$ generates a dense ideal in $A^{-\infty}$, or in other words, it is cyclic in $A^{-\infty}$. Does the converse hold, that is, if $f \in L_{a}^{2}(\mathbb{D})$ is cyclic in $A^{-\infty}$, must then $f$ be cyclic in $L_{a}^{2}(\mathbb{D})$ ?

It is known (see [69]) that the answer to Problem 11 is yes, if we add the assumption that the function $f$ belong to the Nevanlinna class of holomorphic quotients of bounded analytic functions. This in its turn follows rather easily from the case when $f$ is assumed bounded. Leon Brown and Boris Korenblum [21] have obtained the considerably stronger result that if the function $f$ belongs to a slightly smaller Bergman space $L_{a}^{p}(\mathbb{D}), 2<p<$ $+\infty$, then the cyclicity of $f \in L_{a}^{2}(\mathbb{D})$ in $A^{-\infty}$ implies its cyclicity in $L_{a}^{2}(\mathbb{D})$. If a function $f \in L_{a}^{2}(\mathbb{D})$ satisfies

$$
|f(z)| \geq \varepsilon\left(1-|z|^{2}\right)^{N}, \quad z \in \mathbb{D},
$$

for some positive numbers $\varepsilon, N$, then $f$ is invertible in $A^{-\infty}$, and hence cyclic in $A^{-\infty}$.

PROBLEM 12 (Shapiro) Suppose $f \in L_{a}^{2}(\mathbb{D})$ satisfies (9.1). Must then $f$ be cyclic in $L_{a}^{2}(\mathbb{D})$ ?

This problem was settled in the negative by Borichev and Hedenmalm [20]. The construction involved first finding harmonic functions that grow at an appropriate rate radially, and then forming the zero-free function obtained by harmonic conjugation plus exponentiation. 


\section{The index of an invariant subspace}

The index of an invariant subspace $\mathcal{M}$ is defined to be the codimension of $z \mathcal{M}$ in $\mathcal{M}$, i.e. ind $\mathcal{M}=\operatorname{dim}(\mathcal{M} / z \mathcal{M})$. It is clear that $\{0\}$ is the only subspace of index 0 , and it is easy to see that every nonzero singly generated invariant subspace has index 1. Similarly, nontrivial zero based invariant subspaces have index one. In the converse direction it was shown in $[10]$ that every index 1 invariant subspace of $L_{a}^{2}(\mathbb{D})$ is singly generated by its extremal function. It is somewhat difficult to see that there are invariant subspaces of the Bergman space of arbitrary index. This was first established in [12], and it is closely related to the connection between the invariant subspace problem and the apparent difficulty of characterizing all invariant subspaces of $L_{a}^{2}(\mathbb{D})$ that we have alluded to at the very beginning of this article.

The first explicit example of an invariant subspace with index 2 was constructed in [35]. The author exhibits two zero based invariant subspaces $\mathcal{I}(A)$ and $\mathcal{I}(B)$, the zero sequences $A$ and $B$ being disjoint, which are at a positive angle from each other. That entails that their sum $\mathcal{M}=\mathcal{I}(A)+\mathcal{I}(B)$ is a closed invariant subspace of $L_{a}^{2}(\mathbb{D})$, and this easily implies that $\mathcal{M}$ has index 2 . This result was generalized in [48], where a construction was given of an invariant subspace $\mathcal{M}$ of infinite index. The $\mathcal{M}$ is a span of zero based invariant subspaces $\mathcal{I}\left(A_{n}\right), n=1,2,3, \ldots$, where $A_{n}$ is a certain sequence of regularly spaced points in the unit disc that accumulate nontangentially at every boundary point. An alternate way to construct invariant subspaces of arbitrary index was developed in [19] and [1].

The first example raises the following question:

PROBLEM 13 Determine for which pairs of disjoint zero sequences $A$ and $B$ the closed linear span $\mathcal{M}$ of $\mathcal{I}(A)$ and $\mathcal{I}(B)$ has index 1.

We pose two related problems that are perhaps simpler:

PROBLEM 14 Determine the zero sequences A such that every invariant subspace $\mathcal{M}$ that contains $\mathcal{I}(A)$ has index 1.

PROBLEM 15 Determine which $f \in L_{a}^{2}(\mathbb{D})$ have the property that every invariant subspace containing $f$ has index 1.

For the first two questions, one would hope for answers in terms of geometric properties of the sequences $A$ and $B$, while for the third question it would be nice to obtain an answer in terms of the behavior of $f$ near the unit circle.

The index of an invariant subspace is a natural object of investigation, because it is the existence of invariant subspaces of index larger than 1 that drastically distinguishes the situation in $L_{a}^{2}(\mathbb{D})$ from the Hardy space situation. However, we note that Problems 13 and 14 have equivalent formulations that don't mention the index. In fact, in [35], it was shown that given two disjoint zero sequences $A$ and $B$, we either have that $\mathcal{I}(A)+\mathcal{I}(B)$ is dense in $L_{a}^{2}(\mathbb{D})$, or its closure $\mathcal{M}$ has index 2 . Thus, the first problem is equivalent to the question of which disjoint zero sequences $A$ and $B$ have the property that $\mathcal{I}(A)+\mathcal{I}(\mathcal{B})$ is dense in $L_{a}^{2}(\mathbb{D})$. That is how this question was phrased in the original article. Furthermore and similarly, it follows from the results of [47] that Problem 14 is equivalent to which zero sequences $A$ have the property that each invariant subspace $\mathcal{M}$ that contains $\mathcal{I}(A)$ is again a zero based invariant subspace.

There are some partial results known for these problems. First, it follows from [7] that if $A$ and $B$ are two zero sequences for $L_{a}^{2}(\mathbb{D})$ such that there is a point $z_{0} \in \mathbb{T}$ and a planar neighborhood $U$ of $z_{0}$ for which there are nonzero functions $f \in L_{a}^{2}(\mathbb{D}) \cap L^{s}(U \cap \mathbb{D})$ and $g \in L_{a}^{2}(\mathbb{D}) \cap L^{t}(U \cap \mathbb{D}), 1 / \mathrm{s}+1 / \mathrm{t}=1 / 2$, such that $f$ is zero at the points in $A \cap U$ and 
$g$ is zero at the points in $B \cap U$, then the closed linear span of $\mathcal{I}(A)$ and $\mathcal{I}(\mathcal{B})$ in $L_{a}^{2}(\mathbb{D})$ has index 1 (also see [79]). The above-mentioned example in [35] shows that this result is sharp in the sense that if $\varepsilon>0$, then there are two disjoint zero sequences $A$ and $B$ for $L_{a}^{4-\varepsilon}(\mathbb{D})$ such that the closed linear span of $\mathcal{I}(A)$ and $\mathcal{I}(B)$ in $L_{a}^{2}(\mathbb{D})$ has index 2.

There have been a number of papers studying Problems 14 and 15; see [63, 80, 7, 78, $79,9,11]$. Generally, one can state that any nonzero function in any index 2 invariant subspace must be very irregular near every boundary point. Also, using the results of Section 2 of [11] and the results of [12], one can show that if an index 1 invariant subspace $\mathcal{M}$ is contained in an invariant subspace of index $>1$, then for any $n=2,3, \ldots$ or even $n=+\infty, \mathcal{M}$ is contained in an invariant subspace of index $n$.

The most complete results on Problems 14 and 15 have been obtained in [11], where the majorization function $k_{\mathcal{M}}$ of an invariant subspace $\mathcal{M}$ was introduced to study these types of questions:

$$
k_{\mathcal{M}}(\lambda)=\frac{\sup \{|f(\lambda)|: f \in \mathcal{M},\|f\| \leq 1\}}{\sup \left\{|f(\lambda)|: f \in L_{a}^{2}(\mathbb{D}),\|f\| \leq 1\right\}}, \quad \lambda \in \mathbb{D} .
$$

It is easy to see that if $k_{\lambda}(z)$ denotes the Bergman kernel, and if $P_{\mathcal{M}}$ is the orthogonal projection onto $\mathcal{M}$, then

$$
k_{\mathcal{M}}(\lambda)=\frac{\left\|P_{\mathcal{M}} k_{\lambda}\right\|}{\left\|k_{\lambda}\right\|}, \quad \lambda \in \mathbb{D} .
$$

Thus, $k_{\mathcal{M}}$ is continuous and its values lie between zero and 1 . One checks that the analogous definition for the Hardy space $H^{2}(\mathbb{D})$ yields that the majorization function of an invariant subspace of $H^{2}(\mathbb{D})$ equals the modulus of the classical inner function that generates it. Thus, in that case the majorization function of any nonzero subspace has nontangential limit equal to 1 at almost every boundary point. Perhaps it should not be surprising that for $L_{a}^{2}(\mathbb{D})$ the situation is different, but this is precisely related to the questions about the index.

THEOREM 10.1 (Aleman, Richter, Sundberg) Let $\mathcal{M}$ be an invariant subspace of $L_{a}^{2}(\mathbb{D})$ with ind $\mathcal{M}=1$. Then the following are equivalent:

(a) every invariant subspace $\mathcal{N}$ with $\mathcal{M} \subseteq \mathcal{N}$ has index 1 ,

(b) there is a set $E \subset \mathbb{T}$ of positive measure such that $k_{\mathcal{M}}$ has nontangential limit equal to 1 on $E$,

(c) there are $\varepsilon>0$, a positive angle $\sigma$, and a set $E \subset \mathbb{T}$ of positive measure such that $k_{\mathcal{M}}(\lambda) \geq \varepsilon$ for every $\lambda$ in every Stolz angle with opening $\sigma$ and vertex point in $E$,

(d) there is a set $E \subset \mathbb{T}$ of positive measure such that the extremal function $\varphi$ of $\mathcal{M}$ has nontangential limits a.e. on E.

The equivalence of (a) and (d) resolves Problem 15 for extremal functions $f$. For general functions, the answer to Problem 15 has to be more subtle, because there are functions $f$ which have nontangential limits almost everywhere on $\mathbb{T}$ and such that $f$ is contained in some index 2 invariant subspace (see [11] for such an example). Nevertheless, for many choices of the sequence $A$ in Problem 14 or functions $f$ in Problem 15 one can estimate the corresponding majorization function and hence use Theorem 10.1 to decide whether or not $\mathcal{I}(A)$ or $f$ are contained in an invariant subspace of index $>1$.

For example, let us show that if there exists an open set $U$ such that $U \cap \mathbb{T} \neq \emptyset$, and if $f \in L_{a}^{2}(\mathbb{D}), f \neq 0$ is bounded in $U \cap \mathbb{D}$, then every invariant subspace containing $f$ must have index 1. This result is from [7], but here we want to indicate how the majorization function and Theorem 10.1 come in. Let $\mathcal{M}$ be the smallest invariant subspace that contains $f$. As before we let $k_{\lambda}(z)$ be the Bergman kernel, then since $k_{\lambda}$ is bounded for 
each $\lambda \in \mathbb{D}$ the function $g=k_{\lambda} f /\left\|k_{\lambda} f\right\|$ is a function of unit norm in $\mathcal{M}$. The definition of the majorization function implies that

$$
\frac{|f(\lambda)|}{\frac{\left\|k_{\lambda} f\right\|}{\left\|k_{\lambda}\right\|}}=\frac{|g(\lambda)|}{\left\|k_{\lambda}\right\|} \leq k_{\mathcal{M}}(\lambda) \leq 1 .
$$

Now, the hypothesis on $f$ implies that for almost every $z \in E=U \cap \mathbb{T}, f$ has a nontangential limit $f(z)$. Similarly, it is not difficult to show that the hypothesis implies that

$$
\frac{\left\|k_{\lambda} f\right\|}{\left\|k_{\lambda}\right\|}
$$

converges nontangantially to $|f(z)|$ for almost every $z \in U \cap \mathbb{T}$. Thus, whenever both limits exist and are nonzero, the nontangential limit of $k_{\mathcal{M}}$ must be 1 , and the result follows from the equivalence of (a) and (b) of Theorem 10.1.

We already noted above that this argument cannot be extended to cover all functions in $L_{a}^{2}(\mathbb{D})$ that have nontangential limits on a set $E \subset \mathbb{T}$ of positive measure, but in [11] an estimate on the majorization function is used to show the following theorem:

THEOREM 10.2 (Aleman, Richter, Sundberg) Let $E \subset \mathbb{T}$ be closed, have positive measure, and finite entropy, that is, if $\left\{I_{n}\right\}_{n}$ denotes the complementary arcs of $E$, then

$$
\sum_{n}\left|I_{n}\right| \log \frac{1}{\left|I_{n}\right|}<+\infty .
$$

Let $\Omega_{E} \subset \mathbb{D}$ be the union of all Stolz angles with fixed opening angle $\sigma>0$ and vertices at all points of $E$.

If a function $f \in L_{a}^{2}(\mathbb{D}), f \neq 0$, is bounded in $\Omega_{E}$, then every invariant subspace containing $f$ has index 1.

With regards to Problem 14 it had been known for awhile that if the (unrestricted) accumulation points of $A$ omit an arc in $\mathbb{T}$, then any invariant subspace containing $\mathcal{I}(A)$ has index 1 (see [36]). Furthermore, it easily follows from Theorem 10.1 that if a sequence $A \subset \mathbb{D}$ is dominating for $\mathbb{T}$, that is, if almost every $z \in \mathbb{T}$ can be approached nontangentially by a subsequence of $A$, then $\mathcal{I}(A)$ is contained in an invariant subspace of index $>1$. If the sequence $A$ is interpolating for $L_{a}^{2}(\mathbb{D})$, then the converse to this last statement is true, [11]. However, in [11], a zero sequence $A$ for the Bergman space is constructed such that the set of nontangential limit points of $A$ in $\mathbb{T}$ has measure 0 , yet the majorization function $k_{\mathcal{I}(A)}$ is not bounded below in any Stolz angle, and hence $\mathcal{I}(A)$ is contained in invariant subspaces of high index.

\section{Maximal invariant subspaces}

Let us agree to say that an invariant subspace $I$ in $L_{a}^{2}(\mathbb{D})$ is maximal provided every invariant subspace containing it is either $I$ or the whole space $L_{a}^{2}(\mathbb{D})$. If $I$ is maximal, then $L_{a}^{2}(\mathbb{D}) / I$ is a Hilbert space lacking nontrivial invariant subspaces with respect to the induced operator $z[I]: L_{a}^{2}(\mathbb{D}) / I \rightarrow L_{a}^{2}(\mathbb{D}) / I$, so that if $I$ has codimension larger than 1 (it must then have codimension $+\infty$ ), we would have an operator on infinite dimensional Hilbert space with only trivial invariant subspaces. If $I$ is maximal and has codimension 1 , it has the form

$$
I=\left\{f \in L_{a}^{2}(\mathbb{D}): f(\lambda)=0\right\}
$$

for some $\lambda \in \mathbb{D}$. 
PROBLEM 16 Must every maximal invariant subspace of $L_{a}^{2}(\mathbb{D})$ have codimension 1 ?

This problem was answered in the affirmative in [43]. Much more general results were later obtained by Atzmon [15].

PROBLEM 17 Let $\mathcal{M}$ be an invariant subspace in $L_{a}^{2}(\mathbb{D})$. Suppose $\mathcal{N}$ is another invariant subspace that is contained $\mathcal{M}$, which is maximal with respect to this property. Does it follow that $\mathcal{N}$ has codimension 1 inside $\mathcal{M}$ ?

As we were told already in the introduction, this is a disguised version of the invariant subspace problem in separable Hilbert space. The really hard case is when the invariant subspace $\mathcal{M}$ has infinite index.

\section{Methods based on reproducing kernels}

Recall that reproducing kernel $k$ on a set $X$ is a positive definite function of two variables $k: X \times X \mapsto \mathbb{C}$, that is, $k$ satisfies the inequality

$$
\sum_{i, j=0}^{n} c_{i} \bar{c}_{j} k\left(x_{j}, x_{i}\right) \geq 0
$$

whenever $x_{j} \in X, c_{j} \in \mathbb{C}$, for $j=1, \ldots, n$, and $n$ is a positive integer. Moreover, equality holds in (11.1) if and only if $c_{j}=0$ for all $j=1, \ldots, n$. The kernel $k$ determines (uniquely) a Hilbert space $\mathcal{H}(k)$ of functions on $X$ which is the completion of the finite linear combinations of functions of the form $k(x, \cdot)$ with respect to the norm defined in (12.1).

While the classical theory of reproducing kernels is well known, there is a more recent development that has attracted the attention of many researchers in operator theory and complex analysis. More precisely, it appears that certain algebraic properties of such a kernel have a strong impact on a number of important problems in these areas like interpolation, Beurling-type theorems and even factorization theory.

The most investigated class of reproducing kernels consists of kernels $k$ with the properties that the related kernel $1-1 / k$ is positive definite, and $k(z, 0) \equiv 1$. They are called complete Nevanlinna-Pick kernels, and appeared first in some unpublished work by Agler [4] (see also [5]) in connection with Nevannlina-Pick and Carathéodory interpolation as well as commutant-lifting theorems. Arveson [13] studied the complete Nevanlinna-Pick kernel

$$
k(z, \lambda)=\frac{1}{1-\langle z, \lambda\rangle_{d}}
$$

on the unit ball of $\mathbb{C}^{d}, d=1,2,3, \ldots$, because of its connection to the dilation theory of certain commuting operator tuples (called $d$-contractions); here, $\langle\cdot, \cdot\rangle_{d}$ is the Euclidean inner product of $\mathbb{C}^{d}$. The Halmos-Lax-Beurling theorem can be extended to all Hilbert spaces of analytic functions on the unit ball of $\mathbb{C}^{d}$ with a complete Nevanlinna-Pick reproducing kernel. This is a strong result of McCullough and Trent [60], completed by the work of Greene, Richter, and Sundberg [28].

The simplest interesting examples of Hilbert spaces of analytic functions on the unit disk with a complete Nevanlinna-Pick reproducing kernel are the Hardy space and the weighted Dirichlet spaces $D_{\alpha}, 0 \leq \alpha<1$. They consist of analytic functions $f$ in the unit disk with the property that

$$
\|f\|_{\alpha}^{2}=|f(0)|^{2}+\int_{\mathbb{D}}\left|f^{\prime}(z)\right|^{2}\left(1-|z|^{2}\right)^{\alpha} d S(z)<\infty .
$$


Weighted Dirichlet spaces are dual to the weighted Bergman spaces $L_{a}^{2}\left(\mathbb{D}, \omega_{-\alpha}\right)$ via Cauchy duality. More precisely, if we define the Cauchy dual of $L_{a}^{2}\left(\mathbb{D}, \omega_{-\alpha}\right)$ as the space of all analytic functions $g$ in $\mathbb{D}$ with the property that

$$
f \mapsto \lim _{r \rightarrow 1} \int_{-\pi}^{\pi} f\left(r e^{i \theta}\right) \bar{g}\left(r e^{i \theta}\right) d \theta
$$

defines a bounded linear functional on $L_{a}^{2}\left(\mathbb{D}, \omega_{-\alpha}\right)$, then this space coincides with $D_{\alpha}$. This simple fact continues to hold in much greater generality. For example, if $\mu$ is a positive radial measure carried by the unit disk such that

$$
\mu(\{z: r<|z|<1\})>0
$$

for all $r \in(0,1)$, then

$$
L_{a}^{2}(\mathbb{D}, \mu)=L^{2}(\mu) \cap \mathcal{O}(\mathbb{D})
$$

is a Hilbert space of analytic functions whose Cauchy dual defined as above, is a Hilbert space of analytic functions with a complete Nevannlina-Pick reproducing kernel. This follows by a direct application a classical result (see [5]). The problem is whether this holds true for nonradial measures as well. As usual, we will only consider positive measures $\mu$ carried by $\mathbb{D}$ with the property that given any $z \in \mathbb{D}$, there exists a positive constant $C(z)$ such that the estimate

$$
|p(z)|^{2} \leq C(z) \int_{\mathbb{D}}|p|^{2} d \mu
$$

holds for all plynomials $p$. In addition, we assume that $\mu$ is reproducing for the origin, that is,

$$
\int_{\mathbb{D}} p(z) d \mu(z)=p(0)
$$

holds for all polynomials $p$.

PROBLEM 18 Let $\mu$ be a measure as above and let $P^{2}(\mu)$ be the closure of polynomials in $L^{2}(\mu)$. Is it true that the reproducing kernel of the Cauchy dual of $P^{2}(\mu)$ is a complete Nevanlinna-Pick kernel?

The fact that the measure is reproducing at the origin is equivalent to the fact that the reproducing kernel satisfies $k(0, z)=1$ for all $z$. Shimorin has pointed out to us that without this assumption, there are simple examples of measures $\mu$ such that for the corresponding kernel $k$ of the space $P^{2}(\mu), 1-1 / k$ is not positive definite.

A major breakthrough in the theory of Bergman spaces is the following result that appeared first in $[46,47]$ (see also [76]).

THEOREM 12.1 Let $\omega$ be a logarithmically subharmonic weight on $\mathbb{D}$. Then the reproducing kernel $k$ in $L_{a}^{2}(\mathbb{D}, \omega)$ can be written in the form

$$
k(z, \lambda)=\frac{k(0, \lambda) k(z, 0) k(0,0)^{-1}-\bar{\lambda} z l(z, \lambda)}{(1-\bar{\lambda} z)^{2}}
$$

where $l$ is a positive definite function on $\mathbb{D} \times \mathbb{D}$ [i.e., it satisfies (12.1)].

It turns out (see [47] and [76]) that this particular form of the reproducing kernel is equivalent to the having the inequality

$$
\|z f+g\|^{2} \leq 2\left(\|f\|^{2}+\|z g\|^{2}\right)
$$


for any two functions $f, g$ in the space. This is an important reformulation of Theorem 12.1, because it shows that the reproducing kernel of any invariant subspace of index one in $L_{a}^{2}(\mathbb{D}, \omega)$ has the form $(12.2)$ as well. The fact that $(12.3)$ holds in $L_{a}^{2}(\mathbb{D}, \omega)$ follows by a tricky computation based on Green's formula (see [47]). The converse is true for any Hilbert space $\mathcal{H}(k)$ where the operator $M_{z}$, of multiplication by $z$, is bounded and bounded below. Indeed, by considering the operators

$$
T=\left(M_{z}^{*} M_{z}\right)^{-1} \quad \text { and } \quad R: \mathcal{H}(k) \oplus \mathcal{H}(k) \rightarrow \mathcal{H}(k),
$$

where $R$ is defined by

$$
R(f, g)=M_{z} f+T g,
$$

it follows from (12.3) that $\|R\| \leq \sqrt{2}$. A direct computation then shows that for the positive operator $2-R R^{*}$ we have

$$
2-R R^{*}=2-M_{z} M_{z}^{*}-T T^{*},
$$

and (12.2) follows by letting

$$
l(z, \lambda)=\left\langle 2-M_{z} M_{z}^{*}-T T^{*} k(\cdot, \lambda), k(z, \cdot)\right\rangle
$$

since

$$
M_{z} M_{z}^{*} k(z, \lambda)=\bar{\lambda} z k(z, \lambda)
$$

and

$$
T T^{*} k(z, \lambda)=\frac{k(z, \lambda)-k(0, \lambda) k(z, 0) k(0,0)^{-1}}{\bar{\lambda} z} .
$$

To illustrate the power of this representation, let us apply it in order to estimate the reproducing kernel. Suppose that the kernel $k$ on $\mathbb{D}$ satisfies (12.2). Note, first, that for $z=\lambda$, we get

$$
0 \leq k(\lambda, \lambda)=\frac{|k(0, \lambda)|^{2} k(0,0)^{-1}-|\lambda|^{2} l(\lambda, \lambda)}{\left(1-|\lambda|^{2}\right)^{2}} .
$$

In particular, we have

$$
|\lambda|^{2} l(\lambda, \lambda) \leq \frac{|k(0, \lambda)|^{2}}{k(0,0)},
$$

and by the Cauchy-Schwarz inequality, we deduce that

$$
|\bar{\lambda} z l(z, \lambda)| \leq|\lambda z| l(\lambda, \lambda)^{1 / 2} l(z, z)^{1 / 2} \leq|k(0, \lambda)||k(0, z)| k(0,0)^{-1} .
$$

But then, using again (12.2), we see that for $|\lambda|<1$,

$$
|k(z, \lambda)| \leq 2\left(1-|\lambda|^{2}\right)^{-2}|k(0, \lambda)||k(0, z)| k(0,0)^{-1}
$$

Consequently, $k(\cdot, \lambda) / k(0, \cdot)$ is a bounded analytic function for fixed $\lambda \in \mathbb{D}$. Now assume, in addition, that the operator of multiplcation by $z$ on $\mathcal{H}(k)$, written $\left.M_{z}\right|_{\mathcal{H}(k)}$, is a contraction, and apply the von Neumann inequality to conclude that $k(\cdot, \lambda),|\lambda|<1$ can be approximated by polynomial multiples of $k(0, \cdot)$. Since the linear span of these functions is dense in the space we deduce that $\mathcal{H}(k)$ is generated by its extremal function $k(0, \cdot) k(0,0)^{-1 / 2}$. The argument can be extended with appropriate modifications to invariant subspaces of $L_{a}^{2}(\mathbb{D}, \omega)$ that have index greater than one. For this one needs operator-valued reproducing kernels, but the idea is essentially the same. Thus, one can obtain a Beurling-type theorem for such weighted Bergman spaces. The elegant method presented here is essentially due to McCullough and Richter. 
A further consequence of Theorem 12.1 and the Beurling-type theorem is as follows. Let $\mathcal{M} \subset L_{a}^{2}(\mathbb{D})$ be an invariant subspace with index one, denote by $k^{\mathcal{M}}$ its reproducing kernel, and let

$$
k(z, \lambda)=(1-\bar{\lambda} z)^{-2}
$$

be the original Bergman kernel. Then $k^{\mathcal{M}}$ can be written in the form

$$
k^{\mathcal{M}}(z, \lambda)=\frac{k^{\mathcal{M}}(z, 0) k^{\mathcal{M}}(0, \lambda)}{k^{\mathcal{M}}(0,0)}(1-u(z, \lambda)) k(z, \lambda)
$$

where $u$ is a positive definite function. In other words, the normalized reproducing kernel in $\mathcal{M}$ is obtained from the original kernel $k$ by multiplication by the factor $(1-u)$. The surprizing fact about this identity is that it implies the contractive divisor property of the extremal function for $\mathcal{M}$,

$$
\varphi_{\mathcal{M}}=k^{\mathcal{M}}(0,0)^{-1 / 2} k^{\mathcal{M}}(0, z)
$$

that is, the inequality

$$
\left\|f / \varphi_{\mathcal{M}}\right\| \leq\|f\|, \quad f \in \mathcal{M}
$$

Indeed, to see that division by $\varphi_{\mathcal{M}}$ is a contractive operator from $\mathcal{M}$ into $L_{a}^{2}(\mathbb{D})$, it suffices to note that it is the adjoint of the map $T$ defined on linear combinations of reproducing kernels in $L_{a}^{2}(\mathbb{D})$ by the rule

$$
T\left(\sum_{j} c_{j} k\left(\cdot, \lambda_{j}\right)\right)=\sum_{j} c_{j} \frac{k^{\mathcal{M}}\left(\cdot, \lambda_{j}\right)}{\bar{\varphi}_{\mathcal{M}}\left(\lambda_{j}\right)} .
$$

Now, (12.4) actually states that $I-T^{*} T$ is a positive operator, that is, $T^{*}$ is a contraction.

Which other reproducing kernels share this property? This question has been studied by McCullough and Richter [59]. They essentially show that if (12.4) holds for the simplest choices of $\mathcal{M}$, that is, for

$$
\mathcal{M}=\mathcal{M}_{a}=\{f \in \mathcal{H}(k): f(a)=0\}, \quad a \in \mathbb{D},
$$

then $k$ has the form

$$
k(z, \lambda)=\frac{1}{1-\bar{\psi}(\lambda) \psi(z)(1-v(z, \lambda))},
$$

where $\psi$ is analytic in $\mathbb{D}, \psi(0)=0, \psi^{\prime}(0) \neq 0$, while $v$ is a positive definite kernel with $\mathrm{v}(0,0)=0$. Such reproducing kernels $k$ are called Bergman-type kernels and they can also be characterized by a norm inequality in the space $\mathcal{H}(k)$ that resembles $(12.3)$. McCullough and Richter prove that if $\mathrm{k}$ is a Bergman-type kernel, then (12.4) holds for all index one invariant subspaces of $\mathcal{H}(k)$. Moreover, for any zero-based invariant subspace $\mathcal{I}(A)$ of $\mathcal{H}(k)$ the normalized reproducing kernel for $\mathcal{I}(A)$ is a Bergman-type kernel as well. Since (12.4) implies the contractive divisor property, this last fact implies that the analogue of Theorem 4.1 holds for every space $\mathcal{H}(k)$, where $k$ is a Bergman-type kernel.

The above algebraic relations between reproducing kernels and their projections onto invariant subspaces have important consequences related to Problem 6 . To be more precise, for a set $A \subset \mathbb{D}$, let us denote by

$$
\mathcal{I}_{k}(A)=\{f \in \mathcal{H}(k): f=0 \text { on } A\}
$$

the associated invariant subspace given by zeros, and by $\varphi_{A}$ its canonical zero divisor, that is the normalized reproducing kernel at the origin for this invariant subspace. In [8], 
it is shown that if $k$ is a kernel of Bergman-type, then for any point $a \in \mathbb{D}$ we have that $\varphi_{A \cup\{a\}} / \varphi_{A}$ is bounded in $\mathbb{D}$ and satisfies the inequality

$$
\left|\frac{\varphi_{A \cup\{a\}}(z)}{\varphi_{A}(z)}\right| \leq \frac{2+|\alpha|}{\sqrt{2-|\alpha|^{2}}} \leq 3
$$

If $k$ satisfies (12.2), then the quotient

$$
\frac{\varphi_{A \cup\{a\}}(z)}{\varphi_{A}(z)}
$$

is bounded in $\mathbb{D}$ and satisfies

$$
\operatorname{Re} \frac{(1-\bar{a} z) \varphi_{A \cup\{a\}}(z)}{(z-a) \varphi_{A}(z)}>1, \quad z \in \mathbb{D} .
$$

We should point out here that together with the results proved in [6], this last inequality provides an alternative proof of Theorem 3.1 (see [8]).

Although there is a large overlap between kernels of the type (12.2) and the Bergman type kernels, the two classes are distinct [59]. The Bergman kernel $k(z, \lambda)=(1-\bar{\lambda} z)^{-2}$, or more generally, the reproducing kernel for $L_{a}^{2}\left(\mathbb{D}, \omega_{\alpha}\right)$, with $-1<\alpha \leq 0$, are of Bergman type and satisfy condition (12.2) as well, so that, the above estimates provide sharp bounds from above and below for the functions $\varphi_{A \cup\{a\}} / \varphi_{A}$ in these spaces. The following problem has been suggested to us by Sergeř Shimorin.

PROBLEM 19 Let $\omega$ be a logarithmically subharmonic weight on $\mathbb{D}$ that is reproducing at the origin. Is the reproducing kernel $k$ in $L_{a}^{2}(\mathbb{D}, \omega)$ a Bergman-type kernel?

Shimorin [77] has shown that the answer is affirmative for radial weights $\omega$. For nonradial weights, however, the problem is still open.

\section{References}

[1] E. Abakumov and A. Borichev, Shift invariant subspaces with arbitrary indices in $l^{p}$ spaces, J. Funct. Anal. 188 (2002), 1-26.

[2] A. Abkar, Norm approximation by polynomials in some weighted Bergman spaces. J. Funct. Anal. 191 (2002), no. 2, 224-240.

[3] A. Abkar, H. Hedenmalm, A Riesz representation formula for super-biharmonic functions. Ann. Acad. Sci. Fenn. Math. 26 (2001), no. 2, 305-324.

[4] J. Agler, Interpolation (unpublished manuscript).

[5] J. Agler and J. McCarthy, Complete Nevanlinna-Pick kernels, J. Funct. Anal. 175 (2000), 111-124.

[6] A. Aleman, H. Hedenmalm, S. Richter, C. Sundberg, Curious properties of canonical divisors in weighted Bergman spaces, Entire functions in modern analysis [Boris Levin Memorial Conference] (Tel-Aviv, 1997), 1-10, Israel Math. Conf. Proc., 15, Bar-Ilan Univ., Ramat Gan, 2001.

[7] A. Aleman and S. Richter, Some sufficient conditions for the division property of invariant subspaces in weighted Bergman spaces, J. Funct. Anal. 144 (1997), 542556. 
[8] A. Aleman and S. Richter, Single point extremal functions in Bergman-type spaces, Indiana Univ. Math. J. 51 (2002), 581-605.

[9] A. Aleman, S. Richter, and W. Ross,Pseudocontinuations and the backward shift. Indiana Univ. Math. J. 47 (1998), no. 1, 223-276.

[10] A. Aleman, S. Richter, C. Sundberg, Beurling's theorem for the Bergman space, Acta Math. 177 (1996), 275-310.

[11] A. Aleman, S. Richter, C. Sundberg, The majorization function and the index of invariant subspaces in the Bergman spaces. J. Anal. Math. 86 (2002), 139-182.

[12] C. Apostol, H. Bercovici, C. Foias, C. Pearcy, Invariant subspaces, dilation theory, and the structure of the predual of a dual algebra, I J. Funct. Anal. 63 (1985), 369404.

[13] W. Arveson, Subalgebras of $C^{*}$-algebras, III. Multivariable operator theory. Acta Math. 181 (1998), 159-228.

[14] A. Atzmon, Entire functions, invariant subspaces and Fourier transforms. Proceedings of the Ashkelon Workshop on Complex Function Theory (1996), 37-52, Israel Math. Conf. Proc., 11, Bar-Ilan Univ., Ramat Gan, 1997.

[15] A. Atzmon, Maximal, minimal, and primary invariant subspaces. J. Funct. Anal. 185 (2001), 155-213.

[16] S. Bergman, The kernel function and conformal mapping. Second, revised edition. Mathematical Surveys, No. V. American Mathematical Society, Providence, R.I., 1970.

[17] A. Beurling, On two problems concerning linear transformations in Hilbert space, Acta Math. 81 (1949), 39-255.

[18] G. Bomash, A Blaschke-type product and random zero sets for the Bergman space, Ark. Mat. 30 (1992), 45-60.

[19] A. Borichev, Invariant subspaces of a given index in Banach spaces of analytic functions, J. reine angew. Math. 505 (1998), 23-44.

[20] A. Borichev, H. Hedenmalm, Harmonic functions of maximal growth: invertibility and cyclicity in Bergman spaces. J. Amer. Math. Soc. 10 (1997), 761-796.

[21] L. Brown, B. Korenblum, Cyclic vectors in $A^{-\infty}$, Proc. Amer. Math. Soc. 102 (1988), $137-138$.

[22] P. L. Duren, D. Khavinson, H. S. Shapiro, Extremal functions in invariant subspaces of Bergman spaces. Illinois J. Math. 40 (1996), 202-210.

[23] P. L. Duren, D. Khavinson, H. S. Shapiro, C. Sundberg Contractive zero-divisors in Bergman spaces, Pacific J. Math. 157 (1993), 37-56.

[24] P. L. Duren, D. Khavinson, H. S. Shapiro, C. Sundberg, Invariant subspaces and the biharmonic equation, Michigan Math. J. 41 (1994), 247-259.

[25] M. Engliš, A Loewner-type lemma for weighted biharmonic operators, Pacific J. Math. 179 (1997), 343-353. 
[26] M. Engliš, Weighted biharmonic Green functions for rational weights, Glasgow Math. J. 41 (1999), 239-269.

[27] P. R. Garabedian, A partial differential equation arising in conformal mapping, Pacific J. Math. 1 (1951), 485-524.

[28] D. C. V. Greene, S. Richter, C. Sundberg, The structure of inner multipliers in spaces with complete Nevanlinna-Pick kernels, J. Funct. Anal. (to appear).

[29] J. Hadamard, Euvres de Jacques Hadamard, Vols. 1-4, Editions du Centre National de la Recherche Scientifique, Paris, 1968.

[30] J. Hansbo, Reproducing kernels and contractive divisors in Bergman spaces. Zap. Nauchn. Sem. S.-Peterburg. Otdel. Mat. Inst. Steklov. (POMI) 232 (1996), Issled. po Linein. Oper. i Teor. Funktsii. 24, 174-198, 217; translation in J. Math. Sci. (New York) 92 (1998), 3657-3674 .

[31] V. P. Havin, N. K. Nikolski (editors), Linear and complex analysis, Problem book 3, Part II, Lecture Notes in Mathematics 1574, Springer-Verlag, Berlin, 1994.

[32] W. K. Hayman, On a conjecture of Korenblum. Analysis (Munich) 19 (1999), 195205.

[33] H. Hedenmalm, A factorization theorem for square area-integrable analytic functions, J. reine angew. Math. 422 (1991), 45-68.

[34] H. Hedenmalm, A factorization theorem for a weighted Bergman space, Algebra i Analiz 4 (1992), 167-176. (in modified form) St. Petersburg Math. J. 4 (1993), 163174 .

[35] H. Hedenmalm, An invariant subspace of the Bergman space having the codimension two property, J. reine angew. Math. 443 (1993), 1-9.

[36] H. Hedenmalm, Spectral properties of invariant subspaces in the Bergman space, J. Funct. Anal. 116 (1993), 441-448.

[37] H. Hedenmalm, A factoring theorem for the Bergman space, Bull. London Math. Soc. 26 (1994), 113-126.

[38] H. Hedenmalm, A computation of Green functions for the weighted biharmonic operators $\Delta|z|^{-2 \alpha} \Delta$, with $\alpha>-1$, Duke Math. J. 75 (1994), 51-78.

[39] H. Hedenmalm, Open problems in the function theory of the Bergman space, Festschrift in honour of Lennart Carleson and Yngve Domar (Uppsala, 1993), 153169, Acta Univ. Upsaliensis Skr. Uppsala Univ. C Organ. Hist., 58, Uppsala Univ., Uppsala, 1995.

[40] H. Hedenmalm, Boundary value problems for weighted biharmonic operators, St. Petersburg Math. J. 8 (1997), 661-674.

[41] H. Hedenmalm, Recent progress in the function theory of the Bergman space. Holomorphic spaces (Berkeley, CA, 1995), 35-50, Math. Sci. Res. Inst. Publ., 33, Cambridge Univ. Press, Cambridge, 1998.

[42] H. Hedenmalm, Recent developments in the function theory of the Bergman space. European Congress of Mathematics, Vol. I (Budapest, 1996), 202-217, Progr. Math., 168, Birkhuser, Basel, 1998. 
[43] H. Hedenmalm, Maximal invariant subspaces in the Bergman space, Ark. Mat. 36 (1998), 97-101.

[44] H. Hedenmalm, An off-diagonal estimate of Bergman kernels, J. Math. Pures Appl. 79 (2000), 163-172.

[45] H. Hedenmalm, B. Korenblum, K. Zhu, Theory of Bergman spaces. Graduate Texts in Mathematics, 199. Springer-Verlag, New York, 2000.

[46] H. Hedenmalm, S. Jakobsson, S. Shimorin, A maximum principle à la Hadamard for biharmonic operators with applications to the Bergman spaces, C. R. Acad. Sci. Paris Sér. I Math. 328 (1999), 973-978.

[47] H. Hedenmalm, S. Jakobsson, S. Shimorin, A biharmonic maximum principle for hyperbolic surfaces. J. reine angew. Math. 550 (2002), 25-75.

[48] H. Hedenmalm, S. Richter, K. Seip, Interpolating sequences and invariant subspaces of given index in the Bergman spaces. J. reine angew. Math. 477 (1996), 13-30.

[49] H. Hedenmalm, A. L. Shields, Invariant subspaces in Banach spaces of analytic functions, Michigan Math. J. 37 (1990), 91-104.

[50] H. Hedenmalm, S. Shimorin, Hele-Shaw flow on hyperbolic surfaces. J. Math. Pures Appl. 81 (2002), 187-222.

[51] H. Hedenmalm and K. Zhu, On the failure of optimal factorization for certain weighted Bergman spaces, Complex Variables, Theory Appl. 19 (1992), 165-176.

[52] C. Horowitz, Zeros of functions in the Bergman spaces, Duke Math. J. 41 (1974), 693-710.

[53] C. Horowitz, Factorization theorems for functions in the Bergman spaces, Duke Math. J. 44 (1977), 201-213.

[54] P. Koosis, The logarithmic integral. I. Corrected reprint of the 1988 original. Cambridge Studies in Advanced Mathematics, 12. Cambridge University Press, Cambridge, 1998.

[55] B. Korenblum, An extension of the Nevanlinna theory, Acta Math. 135 (1975), 187219.

[56] B. Korenblum, A Beurling-type theorem, Acta Math. 138 (1977), 265-293.

[57] B. Korenblum, A maximum principle for the Bergman space Publ. Mat. 35 (1991), 479-486.

[58] E. LeBlanc, A probabilistic zero set condition for Bergman spaces, Michigan Math. J. 37 (1990), 427-438.

[59] S. McCullough, S. Richter, Bergman-type reproducing kernels, contractive divisors and dilations, J. Funct. Anal. 190 (2002), 447-480.

[60] S. McCullough, T. Trent, Invariant subspaces and Nevanlinna-Pick kernels, J. Funct. Anal. 178 (2000), 226-249.

[61] S. N. Mergelyan, Weighted approximation by polynomials, American Mathematical Society Translations (series 2) 10 (1958), 59-106. 
[62] B. Muckenhoupt, Weighted norm inequalities for the Hardy maximal function, Trans. Amer. Math. Soc. 165 (1972), 207-226.

[63] S. Richter, Invariant subspaces in Banach spaces of analytic functions, Trans. Amer. Math. Soc. 304 (1987), 585-616.

[64] S. Richter, Unitary equivalence of invariant subspaces of Bergman and Dirichlet spaces. Pacific J. Math. 133 (1988), no. 1, 151-156.

[65] S. Richter, A representation theorem for cyclic analytic two-isometries, Trans. Amer. Math. Soc. 328 (1991), 325-349.

[66] K. Seip, Beurling type density theorems in the unit disk Invent. Math. 113 (1993), 21-39.

[67] K. Seip, On a theorem of Korenblum, Ark. Mat. 32 (1994), 237-243.

[68] K. Seip, On Korenblum's density condition for the zero sequences of $A^{-\alpha}$. J. Anal. Math. 67 (1995), 307-322.

[69] A. L. Shields, Cyclic vectors in Banach spaces of analytic functions, Operators and Function Theory Lancaster, 1984), S. C. Power (editor), NATO Adv. Sci. Inst. Ser. C Math. Phys. Sci., 153, Reidel, Dordrecht, 1985, 315-349.

[70] S. M. Shimorin, Factorization of analytic functions in weighted Bergman spaces (Russian), Algebra i Analiz 5 (1993), 155-177; translation in St. Petersburg Math. J. 5 (1994), 1005-1022.

[71] S. M. Shimorin, On a family of conformally invariant operators, St. Petersburg Math. J. 7 (1996), 287-306.

[72] S. M. Shimorin, The Green function for the weighted biharmonic operator $\Delta(1-$ $\left.|z|^{2}\right)^{-\alpha} \Delta$ and the factorization of analytic functions (Russian), Zap. Nauchn. Sem. S.-Peterburg. Otdel. Mat. Inst. Steklov. (POMI) 222 (1995), Issled. po Linein. Oper. i Teor. Funktsii 23, 203-221.

[73] S. M. Shimorin, Single point extremal functions in weighted Bergman spaces, Nonlinear boundary-value problems and some questions of function theory, J. Math. Sci. 80 (1996), 2349-2356.

[74] S. M. Shimorin, The Green functions for weighted biharmonic operators of the form $\Delta w^{-1} \Delta$ in the unit disk, Some questions of mathematical physics and function theory, J. Math. Sci. (New York) 92 (1998), 4404-4411.

[75] S. M. Shimorin, Approximate spectral synthesis in the Bergman space. Duke Math. J. 101 (2000), no. 1, 1-39.

[76] S. M. Shimorin, Wold-type decompositions and wandering subspaces for operators close to isometries, J. reine angew. Math. 531 (2001), 147-189.

[77] S. M. Shimorin, An integral formula for weighted Bergman reproducing kernels, Complex variables, Theory Appl. (to appear).

[78] J. Thomson, and L. Yang, Invariant subspaces with the codimension one property in $L_{a}^{t}()$. Indiana Univ. Math. J. 44 (1995), 1163-1173.

[79] Z. Wu and L. Yang, The codimension-1 property in Bergman spaces over planar regions. Michigan Math. J. 45 (1998), 369-373. 
[80] L. Yang, Invariant subspaces of the Bergman space and some subnormal operators in $\mathbb{A}_{1} \backslash \mathbb{A}_{2}$. Michigan Math. J. 42 (1995), 301-310.

Alexandru Aleman, Department of Mathematics, Lund University, P. O. Box 118, S-221 00 Lund, SwEDEN

Håkan Hedenmalm, Department of Mathematics, Royal Institute of TechNOLOGY, S-100 44 Stockholm, Sweden

Stefan Richter, Department of Mathematics, University of Tennessee, KnOXVILLE TN 37996-1300, USA

E-mail: aleman@maths.lth.se, haakanh@math.kth.se, richer@novell.math.utk.edu. 